\title{
Systems with Outer Constraints. Gupta-Bleuler Electromagnetism as an Algebraic Field Theory
}

\author{
Hendrik Grundling \\ Department of Mathematics, Research School of Physical Sciences, Australian National University, \\ Canberra Australia
}

\begin{abstract}
Since there are some important systems which have constraints not contained in their field algebras, we develop here in a $C^{*}$-context the algebraic structures of these. The constraints are defined as a group $G$ acting as outer automorphisms on the field algebra $\mathscr{F}, \alpha: G \mapsto$ Aut $\mathscr{F}, \alpha_{G} \not \subset \operatorname{Inn} \mathscr{F}$, and we find that the selection of $G$-invariant states on $\mathscr{F}$ is the same as the selection of states $\omega$ on $M(G \underset{\alpha}{\times} \mathscr{F})$ by $\omega\left(U_{g}\right)=1 \forall g \in G$, where $U_{g} \in M(G \underset{\alpha}{\times} \mathscr{F}) \backslash \mathscr{F}$ are the canonical elements implementing $\alpha_{g}$. These states are taken as the physical states, and this specifies the resulting algebraic structure of the physics in $M(G \underset{\alpha}{\times} \mathscr{F})$, and in particular the maximal constraint free physical algebra $\mathscr{R}$. A nontriviality condition is given for $\mathscr{R}$ to exist, and we extend the notion of a crossed product to deal with a situation where $G$ is not locally compact. This is necessary to deal with the field theoretical aspect of the constraints. Next the $C^{*}$-algebra of the CCR is employed to define the abstract algebraic structure of Gupta-Bleuler electromagnetism in the present framework. The indefinite inner product representation structure is obtained, and this puts Gupta-Bleuler electromagnetism on a rigorous footing. Finally, as a bonus, we find that the algebraic structures just set up, provide a blueprint for constructive quadratic algebraic field theory.
\end{abstract}

\section{Introduction}

A degenerate system is defined as having a nonphysical degree of freedom, and is usually characterised by supplementary conditions, or by the action of a gauge group on it. The physicist has the task of extracting the physical subsystem from such a degenerate one. Indeed, physical information such as boundary conditions or constraints, is often injected into a theory through the use of supplementary conditions, and one could argue (as is done in [3]), that in a Lagrangian framework the field equations should also be imposed as supplementary conditions on the field algebra.

In algebraic field theory, a system is described by a unital $C^{*}$-algebra $\mathscr{F}$ as the field algebra, together with its set of states $\wp$, and hence a supplementary condition can be imposed either on $\mathscr{F}$, or on $\wp$, called respectively algebraic and state 
conditions. We examined in previous papers $[1-3]$ both state and algebraic conditions of types which are known to arise often in practice. A state condition consists generally of the specification of a closed subspace $\wp_{D}$ of $\wp$ as the space of all relevant physical states. Such a selection may involve objects either in or outside of $\mathscr{F}$, called respectively inner and outer constraints. In our previous paper [1], we considered only inner constraints of the form:

$$
\wp_{D}=\{\omega \in \wp \mid \omega(U)=1 \forall U \in \mathscr{U}\},
$$

where $\mathscr{U} \subset \mathscr{F}$ is some specified group of unitaries. The latter set was arrived at from the heuristic supplementary conditions of the form $A|\psi\rangle=0$ which imply $\left\langle\psi\left|e^{i \lambda A}\right| \psi\right\rangle=1$, so on applying some correspondence rule, we obtain $\mathscr{U}$ as the set of objects in $\mathscr{F}$ which corresponds to $\exp i \lambda A$. We developed the algebraic structures flowing from this, and called the method of obtaining the physical algebra from it, the $T$-procedure.

However, we found situations in which it was very difficult to apply the $T$ procedure. Two of such were linear field theories with either nonhermitian constraints or nonlinear constraints, the Gupta-Bleuler form of electromagnetism being an example of the former. This was because the constraints could not be defined by unitary elements in $\mathscr{F}$. Moreover, the abstract situation in which gauge degeneracy is defined through the action of a guage group $\alpha: G \mapsto$ Aut $\mathscr{F}$, the physical states $\wp_{D}$ being the gauge invariant states, was also intractable by $T$-procedure. It is for these reasons, with the main one the treatment of nonlinear constraints, that we develop the theory here of systems with outer constraints. This theory is then applied to the Gupta-Bleuler form of electromagnetism, by imposing its constraint $\chi|\psi\rangle=0$ in the nonlinear form $\left\langle\psi\left|\exp \left(i \lambda \chi^{*} \chi\right)\right| \psi\right\rangle=1$, and so it is an example of the treatment both of systems with nonlinear constraints, and of systems with nonhermitian constraints.

The course of this paper runs as follows. In Sect. 2 we summarise the salient points of the $T$-procedure for reference; in Sect. 3 we set up the general problem of systems with outer constraints, followed by the development of the algebraic structures resulting from it. We touch in Sect. 4 briefly upon the structure of the heuristic Gupta-Bleuler formalism, and describe how it will be set up in the ensuing theory. Then follows in Sect. 5 the promised rigorous reconstruction of GuptaBleuler electromagnetism. We also develop some theorems applicable to any linear boson field theory with constraints, and these also prove the nontriviality of a previous example cf. [1], and give connecting points to other procedures used in the literature for electromagnetism as a $C^{*}$-theory [15].

\section{Inner Constraints: the $T$-Procedure}

In this section we collect the basic algebraic structures associated with systems with state conditions, as developed in $[1,3]$, which is where the interested reader can find the proofs of the statements below. As in [4], assume:

2.1. All physical information of a specified system is contained in the pair $\mathscr{F}, \wp$, where the unital $C^{*}$-algebra $\mathscr{F}$ is the field algebra, and $\wp$ is its set of states.

The degeneracy assumption is: 
2.2. There are two specified unitary groups $\mathscr{U}$ and $\mathscr{V}$ in $\mathscr{F}$, such that $\mathscr{U} \cap \mathscr{V}=\mathbf{1}$, respectively called state and algebraic conditions. All physical information is contained in $\mathscr{F}$ and the set of Dirac states defined by:

$$
\wp_{D}:=\{\omega \in \wp \mid\langle\omega \mid U\rangle=1 \forall U \in \mathscr{U}\} .
$$

Then $\omega \in \wp_{D}$ iff $\langle\omega \mid A U\rangle=\langle\omega \mid A\rangle=\langle\omega \mid U A\rangle \forall U \in \mathscr{U}, \forall A \in \mathscr{F}$, or in terms of $L(U):=U-1: \omega \in \wp_{D}$ iff $\{L(\mathscr{U})\} \subset \operatorname{Ker} \omega$ iff $\mathscr{F}\{L(\mathscr{U})\} \cup\{L(\mathscr{U})\} \mathscr{F} \subset \operatorname{Ker} \omega$. If for each $U \in \mathscr{U}$ we define the automorphism $\alpha_{U}:=\operatorname{Ad} U$, then it is clear from the above that the Dirac states are all $\mathscr{U}$-invariant, $\wp_{D} \subset \wp^{\mathscr{U}}$, i.e. $\left\langle\omega \mid \alpha_{U}(A)\right\rangle=\langle\omega \mid A\rangle \forall U \in \mathscr{U}$, $A \in \mathscr{F}, \omega \in \wp_{D}$.

Theorem 2.3. Let $\mathscr{A}(L)$ be the $C^{*}$-algebra generated by $\{L(\mathscr{U})\}$. Then $\omega \in \wp_{D}$ iff $\mathscr{A}(L)$ $\subset \operatorname{Ker} \omega \operatorname{iff}[\mathscr{A}(L) \mathscr{F} \cup \mathscr{F} \mathscr{A}(L)] \subset \operatorname{Ker} \omega$, where $[\cdot]$ denotes the closed linear space generated by its argument.

Theorem 2.4. $\wp_{D} \neq \emptyset$ iff $1 \notin \mathscr{A}(L)$ iff $1 \notin[\mathscr{A}(L) \mathscr{F} \cup \mathscr{F} \mathscr{A}(L)]$, and in this case $\wp_{D}$ contains pure states.

So our nontriviality assumption is:

2.5. Henceforth assume $1 \notin \mathscr{A}(L)$.

For any set $\Omega \subset \mathscr{F}$, define:

$$
\mathscr{M}_{\mathscr{F}}(\Omega):=\{F \in \mathscr{F} \mid F M \in \Omega \ni M F \forall M \in \Omega\},
$$

hence if $\Omega$ is a $C^{*}$-algebra, then $\mathscr{M}_{\mathscr{F}}(\Omega)$ is the largest $C^{*}$-algebra in $\mathscr{F}$ for which $\Omega$ is a two sided ideal.

Theorem 2.6. Let $\mathscr{N}:=[\mathscr{F} \mathscr{A}(L)], \mathscr{D}:=\mathscr{N} \cap \mathcal{N}^{*}$, then $\mathscr{D}$ is the largest $C^{*}$-algebra annihilated by all the Dirac states, i.e. $\mathscr{D}$ is the unique maximal $C^{*}$-algebra in $\mathscr{K}:=\bigcap\left\{\operatorname{Ker} \omega \mid \omega \in \wp_{D}\right\}$.

Theorem 2.7. $\mathcal{O}:=\{F \in \mathscr{F} \mid[F, H] \in \mathscr{D} \forall H \in \mathscr{D}\}=\mathscr{M}_{\mathscr{F}}(\mathscr{D})$.

Then $1 \notin \mathscr{D}$, and $\mathscr{D}$ is a proper two-sided ideal for $\mathcal{O}$. In [5], Dirac defines his observables as "first-class variables" in an analogous way to the way that $\mathcal{O}$ is here defined, i.e. as the "weak commutant" of the constraints. The observables in quantum theories are traditionally taken to be $\mathscr{A}(L)^{\prime}$.

Define $\mathscr{S}$ to be the largest set such that $\mathscr{A}(L) \mathscr{S} \subset[\mathscr{F} \mathscr{A}(L)]$. Then $\mathbf{1} \in \mathscr{A}(L)^{\prime}$ $\subset \mathscr{S} \cap \mathscr{S}^{*}$.

Theorem 2.8. $\mathscr{D}=\overline{\mathscr{S}^{*} \mathscr{A}(L) \mathscr{S}}$ and $\mathcal{O}=\mathscr{S} \cap \mathscr{S}^{*}$.

Hence $\mathscr{A}(L)^{\prime} \subset \mathcal{O}$, and so we could choose $\mathcal{O}$ even as the set of observable quantities. $\mathcal{O}$ can be considered as the largest $C^{*}$-algebra on which we can consistently impose the constraints. Define the maximal $C^{*}$-algebra of physical observables as

$$
\mathscr{R}:=\mathcal{O} / \mathscr{D} \text {. }
$$

The factoring procedure is the actual step of imposing the constraints. Now it is possible that $\mathscr{R}$ may not be simple, and this would not be acceptable for a physical algebra. So, using physical arguments, one would in practice choose a $C^{*}$ subalgebra $\mathcal{O}_{c} \subseteq \mathcal{O}$ containing $\mathscr{A}(L)^{\prime}$ such that

$$
\mathscr{R}_{c}:=\mathcal{O O}_{c} /\left(\mathscr{D} \cap \mathcal{O}_{c}\right) \subset \mathscr{R}
$$


is simple, and then $\mathscr{R}_{c}$ is the right physical algebra. The distinction between $\mathcal{O}$ and $\mathcal{O}_{c}$ was not made in [1]. We call the procedure for obtaining the objects above the $T$ procedure.

For future use, we prove that $\mathcal{O}$ consists of the "weakly gauge invariant elements."

Theorem 2.9. $A \in \mathcal{O}$ iff $\alpha_{U}(A)-A \in \mathscr{D} \forall U \in \mathscr{U}$, i.e. $\left(\alpha_{\mathscr{U}}-l\right) A \subset \mathscr{D}$.

Proof. $L(U) A=(U-1) A=\alpha_{U}(A) U-A=\alpha_{U}(A) L(U)+\left(\alpha_{U}(A)-A\right)$. Now $A \in \mathscr{S}$ iff $L(\mathscr{U}) A \subset[\mathscr{F} \mathscr{A}(L)]$, i.e. by the first relation, $A \in \mathscr{S} \operatorname{iff}\left(\alpha_{L}(A)-A\right) \in$ $[\mathscr{F} \mathscr{A}(L)] \forall U \in \mathscr{U}$. On using the adjoint of the latter expression, we get from 2.8 that $\left(\alpha_{\mathscr{O}}-l\right) A \subset \mathscr{D}$ iff $A \in \mathcal{O}$.

Theorem 2.10. $\omega \in \wp_{D}$ iff $\pi_{\omega}(\mathscr{D}) \Omega_{\omega}=0$, where $\pi_{\omega}$ and $\Omega_{\omega}$ are respectively the GNSrepresentation of $\omega$ and its cyclic vector.

This corresponds to the heuristic $\chi|\psi\rangle=0$ method for imposing constraints. Define $\boldsymbol{r}:=\{\alpha \in$ Aut $\mathscr{F} \mid \mathscr{D}=\alpha[\mathscr{D}]\}$, then since $\mathcal{O}=\mathscr{M}_{\mathscr{F}}(\mathscr{D}), \alpha$ also preserves $\mathcal{O}$ and so defines canonically an automorphism $\alpha^{\prime}$ on $\mathscr{R}$. Define the group homomorphism $T: \mathbf{r} \mapsto$ Aut $\mathscr{R}$ by $T(\alpha)=\alpha^{\prime}$, then we expect Ker $T$ to consist of gauge transformations:

Theorem 2.11. Ker $T=\left\{\alpha \in\right.$ Aut $\mathscr{F} \mid\langle\omega \mid \alpha[A] F\rangle=\langle\omega \mid A F\rangle \forall A, F \in \mathcal{O}$ and $\left.\forall \omega \in \wp_{D}\right\}$ $\subset \mathbf{r}$.

Theorem 2.12. $\alpha \in \operatorname{Inn} \mathscr{F} \cap \boldsymbol{\gamma} \Rightarrow \alpha^{\prime} \in \operatorname{Inn} \mathscr{R}$.

The physically admissable automorphisms of $\mathscr{F}$ denoted by $\mathbf{\Upsilon}_{c}$, are those which are definable on $\mathscr{R}_{c}$, i.e. $\alpha\left(\mathcal{O}_{c}\right)=\mathcal{O}_{c}$, and $\alpha\left(\mathscr{D} \cap \mathcal{O}_{c}\right) \subseteq \mathscr{D} \cap \mathcal{O}_{c}$. Clearly, if $\alpha \in \mathbf{Y}$. it is sufficient that it satisfies $\alpha\left(\mathcal{O}_{c}\right) \subseteq \mathcal{O}_{c}$ for it to be physically admissable.

Next consider the algebraic conditions $\mathscr{V}$. Define $N(V):=V-\mathbf{1}, V \in \mathscr{V}$. It is hard to find an abstract interpretation of the heuristic condition $N(\mathscr{V})=0$. We interpreted it previously [3], to mean either that by construction of $\mathscr{F}$ the abstract object that would have corresponded to $N(V)$ is identically zero (cf. [6] for an example of this approach), or to mean that there is some ${ }^{*}$-homomorphism $\Gamma: \mathscr{P}$ $\subset \mathscr{F} \mapsto \mathscr{R}_{a}$ onto, with $N(\mathscr{V}) \subset \operatorname{Ker} \Gamma$. Clearly in this case $\mathscr{R}_{a}=\mathscr{P} / \operatorname{Ker} \Gamma$. Now if $\Gamma$ is not the $T$-procedure above, there are ordering problems in systems where both types of constraints need to be imposed, and so the natural conclusion is that the two best options for dealing with algebraic conditions are:

(i) Construct $\mathscr{F}$ in such a way that the objects in it which correspond to the heuristic constraints are identically zero, or

(ii) treat all constraints on the same footing, i.e. impose them according to the $T$ procedure.

Finally, for later reference we sketch the structures found for a linear boson field with linear hermitian constraints, such as the Dirac form of electromagnetism, cf. [1]. The field algebra is taken as Manuceau's $C^{*}$-algebra of the CCR cf. $[4,7]: \mathscr{F}=\overline{\Delta(\mathscr{M}, B)}$, where $\mathscr{M}$ is the complexified test function space with the nondegenerate symplectic form $B(\cdot, \cdot)$ on it. To fix notation for the later sections, we define $\overline{\Delta(\mathscr{M}, B)}$.

Definition 2.13. (i) Given a linear topological space $\mathscr{M}$ with symplectic form $B$ on it, 
let $\Delta(\mathscr{M}, B)$ be the normed *-algebra such that its elements are the complex valued functions on $\mathscr{M}$ with finite support. It has the obvious linear structure, the following multiplication law:

$$
\left(f_{1} f_{2}\right)(z):=\sum_{z_{1} \in \mathscr{M}} f_{1}\left(z_{1}\right) f_{2}\left(z-z_{1}\right) \exp \left[-i B\left(z_{1}, z\right) / 2\right]
$$

involution $f^{*}(z):=\overline{f(-z)}$ and the norm $\|f\|_{1}:=\sum_{z \in \mathscr{M}}|f(z)|$.

(ii) Denote the completion of $\Delta(\mathscr{M}, B)$ in the latter norm by $\Delta_{1}(\mathscr{M}, B)$. Then $\overline{\Delta(\mathscr{M}, B)}$ is the enveloping $C^{*}$-algebra of $\Delta_{1}(\mathscr{M}, B)$, i.e. the closure in the enveloping $C^{*}$-norm: $\|f\|:=\sup _{\pi \in P}\|\pi(f)\|$, where $P$ is the set of nondegenerate representations of $\Delta_{1}(\mathscr{M}, B)$.

The functions $\delta_{F}$ of support $\{F\}$ and value 1 form a generating set of unitaries for $\mathscr{F}$. The heuristic correspondence rule is $\delta_{F} \leftrightarrow W(F)$, where $W(F)$ is the heuristic Weyl operator constructed from the smeared version of a set of canonical pairs $p_{i}(\underline{x}), q_{i}(\underline{x})$. The constraint group $\mathscr{U}$ is then specified as the group generated by $\delta_{\mathscr{C}}:=\left\{\delta_{F} \mid F \in \mathscr{C}\right\}$, where $\mathscr{C}$ is a real linear subspace of $\mathscr{M}$ corresponding to the heuristic constraints. With

$$
\mathbf{p}:=\{H \in \mathscr{M} \mid B(H, C)=0 \forall C \in \mathscr{C}\},
$$

we found that $\delta_{\mathbf{p}}=\delta_{\mathscr{M}} \cap \mathcal{O}$, and indeed $C^{*}\left(\delta_{\mathbf{p}}\right)=C^{*}\left(\delta_{\mathscr{M}} \cap \mathcal{O}\right)=\mathscr{A}(L)^{\prime}$. There are additional elements to these in $\mathcal{O}$, of the form $\sum_{i} \alpha_{i} \delta_{F_{r}}$ with $F_{i} \notin \mathbf{p} \forall i$, but it is very difficult to get our hands on these, and so we make the choice: $\mathcal{O}_{c}=C^{*}\left(\delta_{\mathrm{p}}\right)=\mathscr{A}(L)^{\prime}$. Now $\mathscr{A}(L) \backslash \mathcal{O}_{c}$ does not affect $\mathscr{R}_{c}$, and hence we might as well require $\mathscr{A}(L) \subset \mathcal{O}_{c}$, i.e. $\mathscr{C} \subset \mathbf{p}$, so in this case $\mathscr{A}(L)$ is commutative, and $\mathscr{U}=\delta_{\mathscr{L}}$. Then $\mathscr{D} \cap \mathscr{O}_{c}=\mathscr{A}(L) C^{*}\left(\delta_{\mathbf{p}}\right)$, and the physical algebra is $\mathscr{R}_{c}=\mathscr{A}(L)^{\prime} / \overline{\mathscr{A}(L)^{\prime} \mathscr{A}(L)}$. In Sect. 5 of this paper we show that when $\mathscr{C}$ is the degenerate part of $\mathbf{p}$ with respect to $B$, then $\mathscr{R}_{c}=$ $\overline{\Delta(\mathbf{p} / \mathscr{C}, \widetilde{B})}$, where $\widetilde{B}$ is the canonical image of $B$ on $\mathbf{p} / \mathscr{C}$ (which is nondegenerate), and so in this case $\mathscr{R}_{c}$ is simple. The symplectic transformations on $\mathscr{M}$ which conserve $\mathscr{C}$ will define automorphisms on $\mathscr{R}_{c}$.

In [2] we exhibited the connection between the structures above, and the usual structures of indefinite inner product representations, but we omit a discussion of that here, since it will not be utilized.

\section{General Abstract Systems with Outer Constraints}

To set up the problem of outer constraints, consider two typical situations. First, consider a concrete situation that occurs often in physics. Let the field algebra $\mathscr{F}$ be concrete on a Hilbert space $\mathscr{H}$, and let there be given a constraint $\chi \in \mathrm{Op}(\mathscr{H})$, where Op $(\mathscr{H})$ denotes the set of (bounded and unbounded) operators with their domains and ranges in $\mathscr{H}$. The physical subspace is selected by $\mathscr{H}_{p}:=\{\psi \in \mathscr{H} \mid$ $\chi \psi=0\}$, the physical representation space is $\overline{\mathscr{H}}_{p}$, and the physical representation is simply restriction to $\mathscr{H}_{p}$ and closure. The physical observable algebra $\mathcal{O}$ consists of all elements for which this representation $\pi$ makes sense, and this includes the commutant of $\chi$, and consists of all elements which preserve $\overline{\mathscr{H}}_{p}$. The physical 
algebra is $\pi(\mathcal{O})$, i.e. all elements which are zero on $\mathscr{H}_{p}$ have been factored out. In the case when $\chi$ is hermitian (respectively nonhermitian), we can construct the unitaries $U_{\lambda}:=\exp i \lambda \chi\left(\right.$ respectively $\left.U_{\lambda}:=\exp i \lambda \chi^{*} \chi\right)$. Then $\left(\psi, U_{\lambda} \psi\right)=1$ will be an equivalent selection condition for $\mathscr{H}_{p}$. If $U_{\lambda} \in \mathscr{F}$, we have the case of inner constraints, and so, since this is done already, consider the case $U_{\lambda} \notin \mathscr{F}$. In this case we can extend the field algebra $\mathscr{F}$ to the $C^{*}$-algebra $\mathscr{F}_{e}:=C^{*}\left(\mathscr{F} \cup U_{\mathbb{R}}\right)$, execute the $T$-procedure on $U_{\mathbb{R}}$ in $\mathscr{F}_{e}$, and intersect the resultant algebraic structures $\mathscr{D}_{e}, \mathcal{O}_{e}$ with $\mathscr{F}$. This will correspond to the selection of states $\omega$ on $\mathscr{F}_{e}$ by $\omega\left(U_{\mathbb{R}}\right)=1$, and restriction to $\mathscr{F}$. This is in the spirit of what we intend to do later.

Second, we wish to consider the situation where an abstract field $C^{*}$-algebra $\mathscr{F}$ is given, and the constraints can only be specified as automorphisms on $\mathscr{F}$, that is, there is specified an action of a locally compact group $G$ on $\mathscr{F}$, i.e. $\alpha: G \mapsto$ Aut $\mathscr{F}$. Locally compactness, though essential for the definition of $G \underset{\alpha}{\times} \mathscr{F}$, will be relaxed later, cf. Sect. 5. In this situation, it seems that the only available selection criterion for physical states is $G$-invariance, taking $\wp^{G}(\mathscr{F})$ as the physical states. This is because even if $\alpha_{G}$ is inner, there is generally not a unique unitary group homomorphism $U: G \mapsto \mathscr{F}_{u}$ which implements $\alpha$ by $\alpha_{g}=\operatorname{Ad} U_{g}$. The nonuniqueness originates from the fact that Inn $\mathscr{F} \cong \mathscr{F}_{u} / Z\left(\mathscr{F}_{u}\right)$, and hence given a $U: G \mapsto \mathscr{F}_{u}$ as above, and a homomorphism $\lambda: G \mapsto Z\left(\mathscr{F}_{u}\right)$, then we find $\widetilde{U}: G \mapsto \mathscr{F}_{u}$ defined by $\tilde{U}_{g}:=U_{g} \lambda_{g}$ will also implement $\alpha, \alpha_{g}=\operatorname{Ad} U_{g}=\operatorname{Ad} \widetilde{U}_{g}$. So even if $\alpha_{G} \subset \operatorname{Inn} \mathscr{F}$, we cannot choose a $U: G \mapsto \mathscr{F}_{u}$ such that $\alpha=\operatorname{Ad} U$, and then impose the $T$-procedure on $U_{G}$. Information is lost in specifying a constraint as an automorphism instead of a unitary element. This situation is clearly embedded in the inner constraint situation of Sect. 2, where we saw that $\wp_{D} \subset \wp^{\mathscr{U}}\left(=\wp^{G}\right)$, i.e. that the selection condition $\omega(U)$ $=1$ is stronger than $\omega\left(U A U^{-1}\right)=\omega(A) \forall A \in \mathscr{F}$. The general theory which is developed in this section is independent of whether the constraint automorphism group is inner or not, but for physical reasons, we want at least a generating set of $G$ to be outer. If otherwise, we should still be looking for a more unique way to define the constraints in $\mathscr{F}$. Henceforth this will be called the outer constraint situation.

On constructing the $C^{*}$-algebra $\mathscr{F}_{e}:=M(G \underset{\alpha}{\times} \mathscr{F})$, we know that there is an identification such that $\mathscr{F} \subset \mathscr{F}_{e}$, cf. [8]. Moreover, there are unitaries $U_{g}$ satisfying $\alpha_{g}=\operatorname{Ad} U_{g}$, and $U_{H} \subset \mathscr{F}{ }_{e} \backslash \mathscr{F}$ for $H$ a generating subset of $G$. Since the unitaries $U_{G}$ are canonically and uniquely defined, we may try to apply the $T$-procedure in $\mathscr{F}_{e}$ to $U_{G}$, i.e. to select the physical states on $\mathscr{F}_{e}$ by $\omega\left(U_{G}\right)=1$, and then to restrict these to $\mathscr{F}$. In Theorem 3.3 we prove that this selects exactly the set of $G$-invariant states $\wp^{G}(\mathscr{F})$ on $\mathscr{F}$. It is tempting despite the uniqueness problem, to identify $U_{g}$ with the heuristic object $U_{\lambda}$ of the concrete situation above, if $\operatorname{Ad} U_{\lambda} \in$ Aut $\mathscr{F}$. Hence we set up the problem of outer constraints by:

Assumption 3.1. In addition to Assumption 2.1, we have the action of a locally compact gauge group $\alpha: G \mapsto$ Aut $\mathscr{F}$ such that $\alpha(G) \not \subset$ Inn $\mathscr{F}$. Then the physical states on $\mathscr{F}$ are defined by $\wp_{D}:=\wp_{D e} \mid \mathscr{F}$, where

$$
\wp_{D e}:=\left\{\omega \in \wp\left(\mathscr{F}_{e}\right) \mid \omega\left(U_{g}\right)=1 \forall g \in G\right\} .
$$

We develop the algebraic structures resulting from 3.1. The specification of $\wp_{D e}$ starts the $T$-procedure in $\mathscr{F}_{e}: L_{g}:=U_{g}-\mathbf{1}, \mathscr{A}(L):=C^{*}\left(L_{G}\right), \mathscr{D}_{e}:=\left[\mathscr{F}_{e} \mathscr{A}(L)\right] \cap$ 
$\left[\mathscr{A}(L) \mathscr{F}_{e}\right], \quad \mathscr{O}_{e}:=\mathscr{M}_{\mathscr{F}_{e}}\left(\mathscr{D}_{e}\right) \equiv$ multiplier algebra of $\mathscr{D}_{e}$ in $\widetilde{F}_{e}$. The nontriviality requirement is $\mathscr{A}(L) \not \mathbf{1}$, and $\mathscr{D}_{e}$ is the largest $C^{*}$-algebra in $\mathscr{F}_{e}$ annihilated by all $\omega \in \wp_{D e}$. Then the $C^{*}$-algebra $\mathscr{D}:=\mathscr{F} \cap \mathscr{D}_{e}$ is the largest $C^{*}$ algebra in $\mathscr{F}$ annihilated by all $\omega \in \wp_{D}$, because $\wp_{D}=\wp_{D e} \mid \mathscr{F}$. Moreover, $\wp_{D}$ is the full set of states on $\mathscr{F}$ which vanish on $\mathscr{D}$, because any such a state can be extended to a state on $\mathscr{F}_{e}$ which vanishes on $\mathscr{D}_{e}$. This leads one to ask whether we can now ignore objects external to $\mathscr{F}$, and carry the $T$-procedure through on $\mathscr{D}$ in $\mathscr{F}$, i.e. construct $\mathcal{O}:=\mathscr{M}_{\mathscr{F}}(\mathscr{D})$ and set $\mathscr{R}_{1}:=\mathscr{O} / \mathscr{D}$ as the maximal physical algebra? The answer is no, because $\mathscr{R}_{1}$ will not be a gauge invariant algebra, and we see this as follows. Since $\mathscr{D}_{e} \triangleleft \mathcal{O}_{e}$, and $\mathscr{F}$ is a $C^{*}$-algebra, $\left(\mathscr{D}_{e} \cap \mathscr{F}\right) \triangleleft\left(\mathcal{O}_{e} \cap \mathscr{F}\right)$, and hence $\mathcal{O}_{e} \cap \mathscr{F} \subset \mathscr{M}_{\mathscr{F}}(\mathscr{D})=\mathcal{O}$. This inclusion is usually proper (seen through examples, cf. Sect. 5) except if $\mathscr{A}(L) \subset \mathscr{F}$ (cf. 3.2 below), which is the inner constraint situation. Hence for outer constraints, the restriction of the $T$-procedure of $\mathscr{F}_{e}$ to $\mathscr{F}$, results in a smaller algebra than the corresponding $T$-procedure in $\mathscr{F}$. For $\mathscr{R}_{1}$ to be gauge invariant, the gauge transformations must be definable and trivial on it, i.e. $\alpha_{g}=\operatorname{Ad} U_{g}$ must preserve $\mathscr{D}$ and each equivalence class of $\mathcal{O}$. By a previous theorem (cf. 3.7 in [1]), $\alpha_{g}$ preserves $\mathscr{D}_{e}$, by definition preserves $\mathscr{F}$, and hence preserves $\mathscr{D}$. For $\alpha_{g}$ to be the identity on $\mathscr{R}$, we need $\alpha_{g} \in \operatorname{Ker} T$, i.e. $\left(\alpha_{G}-l\right) \mathcal{O} \subset \mathscr{D}$. Whilst it is always true that $\left(\alpha_{G}-\imath\right) \mathcal{O}_{e} \subset \mathscr{D}_{e}$, cf. 2.9 , and hence $\left(\alpha_{G}-\imath\right)\left(\mathcal{O}_{e} \cap \mathscr{F}\right) \subset\left(\mathscr{D}_{e} \cap \mathscr{F}\right)=\mathscr{D}$, there is no way to ensure this for the additional elements in $\mathcal{O}$. Hence in general $\mathscr{R}_{1}$ is not gauge invariant. The true restriction $\mathscr{R}:=\left(\mathcal{O}_{e} \cap \mathscr{F}\right) /\left(\mathscr{D}{ }_{e} \cap \mathscr{F}\right)$ will be the proper choice for the maximal physical algebra, because it is gauge invariant and constraint free, and this choice is justified because $\mathcal{O}_{e} \cap \mathscr{F}$ contains the gauge invariant elements $\mathscr{A}(L)^{\prime} \cap \mathscr{F}$, which are the usual observables. Since $\alpha_{g}$ preserves $\mathscr{F}$, Theorem 2.9 provides an internal characterization of $\mathcal{O}_{e} \cap \mathscr{F}$, i.e. given $\mathscr{D}$ and $\mathscr{F}, A \in \mathcal{O}_{e} \cap \mathscr{F}$ iff $\left(\alpha_{G}-\imath\right) A \subset \mathscr{D}$.

Next we wish to examine how sensitive the previous construction is to the choice of $\mathscr{F}_{e}$. This is because it seems equally plausible to have chosen $\mathscr{F}_{e}=C^{*}\left(\mathscr{F} \cup U_{G}\right)$ instead of $M(G \underset{\alpha}{\times} \mathscr{F})$, or any other $C^{*}$-algebra in $M(G \underset{\alpha}{\times} \mathscr{F})$ which contains $\mathscr{F} \cup U_{G}$. Hence we consider the problem of extending or restricting degenerate systems.

Theorem 3.2. Let $\mathscr{F} \subset \mathscr{F}_{e}$ be two unital $C^{*}$-algebras and let a constraint algebra be specified: $\mathscr{A}(L) \subset \mathscr{F}$. Execute the T-procedure in both $\mathscr{F}$ and $\mathscr{F}_{e}$, and denote the corresponding objects in the latter by the subscript ' $e$ '. Then

(i) $\mathscr{D}_{e} \cap \mathscr{F}=[\mathscr{F} \mathscr{A}(L)] \cap[\mathscr{A}(L) \mathscr{F}]=\mathscr{D}$,

(ii) $\mathcal{O}_{e} \cap \mathscr{F}=\mathscr{M}_{\mathscr{F}}(\mathscr{D})=\mathcal{O}$,

and hence $\mathscr{R}=\mathcal{O} / \mathscr{D}=\left(\mathcal{O}_{e} \cap \mathscr{F}\right) /\left(\mathscr{D}_{e} \cap \mathscr{F}\right)$.

Proof. (i) $\mathscr{A}(L) \subset \mathscr{F}$ means that $\wp_{D}(\mathscr{F}):=\wp_{D}\left(\mathscr{F}_{e}\right) \mid \mathscr{F}$, and conversely, all extensions $\tilde{\omega}$ of $\omega \in \wp_{D}(\mathscr{F})$ are in $\wp_{D}\left(\mathscr{F}_{e}\right)$. Generally, if $\omega \in \wp(\mathscr{F}), \tilde{\omega} \in \wp(\mathscr{F} e), \tilde{\omega} \mid \mathscr{F}=\omega$, then $N_{\omega}=N_{\dot{\omega}} \cap \mathscr{F}$, where $N_{\omega}$ is the left kernel of $\omega$. Hence:

$$
\bigcap\left\{N_{\omega} \mid \omega \in \wp_{D}(\mathscr{F})\right\}=\bigcap\left\{N_{\tilde{\omega}} \mid \tilde{\omega} \in \wp_{D}\left(\mathscr{F}_{e}\right)\right\} \cap \mathscr{F},
$$

and so by Theorem $2.12[1]:[\mathscr{F} \mathscr{A}(L)]=\left[\mathscr{F}_{e} \mathscr{A}(L)\right] \cap \mathscr{F}$. Then $\mathscr{D}_{e} \cap \mathscr{F}=$ $\left[\mathscr{F}_{e} \mathscr{A}(L)\right] \cap\left[\mathscr{A}(L) \mathscr{F}_{e}\right] \cap \mathscr{F}=[\mathscr{F} \mathscr{A}(L)] \cap[\mathscr{A}(L) \mathscr{F}]=\mathscr{D}$. 
(ii) $\mathscr{S}_{e}$ is the largest set in $\mathscr{F}_{e}$ such that $\mathscr{A}(L) \mathscr{S}_{e} \subset\left[\mathscr{F}_{e} \mathscr{A}(L)\right]$, and hence $\mathscr{S}_{e} \cap \mathscr{F}$ is the largest set in $\mathscr{F}$ such that $\mathscr{A}(L)\left(\mathscr{S}_{e} \cap \mathscr{F}\right) \subset\left[\mathscr{F}_{e} \mathscr{A}(L)\right]$. Now as $\mathscr{F}$ is a $C^{*}$-algebra, $\mathscr{A}(L)\left(\mathscr{S}_{e} \cap \mathscr{F}\right) \subset \mathscr{F}$, i.e. $\mathscr{A}(L)\left(\mathscr{S}_{e} \cap \mathscr{F}\right) \subset\left[\mathscr{F} e_{e} \mathscr{A}(L)\right] \cap \mathscr{F}=[\mathscr{F} \mathscr{A}(L)]$. Hence $\mathscr{S}=\mathscr{S}_{e} \cap \mathscr{F}$ and so $\mathcal{U}_{e}=\mathscr{S}_{e} \cap \mathscr{S}_{e}^{*}$ implies that $\mathcal{O}_{e} \cap \mathscr{F}=\mathscr{S} \cap \mathscr{S}^{*}=$ $\mathcal{O}=\mathscr{M}_{\mathscr{F}}(\mathscr{D})$.

So the preceding $T$-procedure would be unaffected by the choice of $\mathscr{F}_{e}$ in $M(G \underset{\alpha}{\times} \mathscr{F})$, as long as it contains both $\mathscr{F}$ and $U_{G}$.

In the next Theorem 3.3, we will show that the restriction of the Dirac states of $\mathscr{F}_{e}$ to $\mathscr{F}$ is the same as the set of $G$-invariant states of $\mathscr{F}$, i.e. the $T$-procedure on $\mathscr{F}_{e}$, restricted to $\mathscr{F}$ only results in the structures arising from the selection of the gauge invariant states on $\mathscr{F}$.

First, to fix notation, we define the crossed product $G \times \underset{\alpha}{\times} \mathscr{F}$ cf. $[8,9]$. Let there be given an action $\alpha: G \mapsto$ Aut $\mathscr{F}$, where $G$ is a locally compact group, with a left Haar measure $\lambda$, and the modular function $\Delta: G \mapsto \mathbb{R}$ is a homomorphism. Let $L^{1}(G, \mathscr{F})$ denote the Bochner integrable functions $f: G \mapsto \mathscr{F}$ with relation to the symmetric Haar measure: $d s:=\Delta(s)^{-1 / 2} d \lambda(s)$. This is a Banach *algebra with the following operations:

$$
(f * g)(r):=\int f(s) \alpha_{s}\left(g\left(s^{-1} r\right)\right) d \lambda(s), \quad f^{*}(r):=\alpha_{r}\left(f\left(r^{-1}\right)^{*}\right) .
$$

Then $G \underset{\alpha}{\times} \mathscr{F}$ is the enveloping $C^{*}$-algebra of $L^{1}(G, \mathscr{F})$. The actions of $G$ and $\mathscr{F}$ on $L^{1}(G, \mathscr{F})$ defined respectively by:

$$
(U(s) f)(r):=\Delta(s)^{1 / 2} \alpha_{s}\left(f\left(s^{-1} r\right)\right) \text { and }(\pi(A) f)(r):=A f(r)
$$

extend to actions on $G \underset{x}{\times} \mathscr{F}$, and induce the homomorphisms

$$
U: G \mapsto M(G \underset{\alpha}{\times} \mathscr{F}) \text { and } \pi: \mathscr{F} \mapsto M(G \underset{\alpha}{\times} \mathscr{F})
$$

which are taken as identifications, [8]. The notation $M(\cdot)$ used here, refers to the abstract multiplier algebra of the argument, cf. [19] and [8]. Then $\alpha_{s}=\operatorname{Ad} U(s)$, and $L^{1}(G, \mathscr{F})$ has an approximate identity. Revert to the previous notation, then it is in the sense above that we say $\widetilde{\mathscr{F}} \subset \mathscr{F}_{e}$.

Theorem 3.3. $\wp_{D}(M(G \underset{\alpha}{\times} \mathscr{F})) \mid \mathscr{F}=\wp^{G}(\mathscr{F}):=\left\{\omega \in \wp(\mathscr{F}) \mid \omega\left(\alpha_{g}(A)\right)=\omega(A) \forall g \in G\right.$, $A \in \mathscr{F}\}$.

Proof. Clearly by definition $\wp_{D}\left(\mathscr{F}_{e}\right) \mid \mathscr{F} \subseteq \wp^{G}(\mathscr{F})$, cf. remark above 2.3. For equality we need to show that each $\omega \in \wp^{G}(\mathscr{F})$ has an extension $\tilde{\omega} \in \wp_{D}\left(\mathscr{F}_{e}\right)$, $\tilde{\omega} \mid \mathscr{F}=\omega$. Let $\omega \in \wp^{G}(\mathscr{F})$, and construct $\tilde{\omega}$ as follows. For $f \in L^{1}(G, \mathscr{F})$,

$$
\tilde{\omega}(f):=\int \omega(f(r)) \Delta(r)^{-1 / 2} d \lambda(r)=\int \omega(f(r)) d r .
$$

Check that it is a state:

$$
\begin{aligned}
\tilde{\omega}\left(f^{*} * f\right) & =\omega\left(\iint \alpha_{r}\left(f\left(r^{-1}\right)^{*}\right) \chi_{r}\left(f\left(r^{-1} s\right)\right) \Delta(s)^{-1 / 2} d \lambda(r) d \lambda(s)\right) \\
& =\omega\left(\iint f\left(r^{-1}\right)^{*} f\left(r^{-1} s\right) \Delta(s)^{-1 / 2} d \lambda(r) d \lambda(s)\right) \\
& =\omega\left(\iint f(r)^{*} f(s) \Delta(r)^{-1 / 2} \Delta(s)^{-1 / 2} d \lambda(r) d \lambda(s)\right) \geqq 0
\end{aligned}
$$


because $\omega$ is a state. Normalisation is simple. Continuity:

$$
|\tilde{\omega}(f)| \leqq \int|\omega(f(r))| \Delta(r)^{-1 / 2} d \lambda(r) \leqq \int\|f(r)\| \Delta(r)^{-1 / 2} d \lambda(r)=\|f\|_{L^{1}} .
$$

Hence $\tilde{\omega}$ is a positive and continuous form on the Banach *algebra $L^{1}(G, \mathscr{F})$, and hence by Dixmier 2.1.5 [10] is hermitian, and so by Dixmier 2.7.5 has a unique $C^{*}$ continuous extension as a state to the $C^{*}$-envelope $G \underset{\alpha}{\times} \mathscr{F}$. This state in turn extends uniquely to a state on $\mathscr{F}_{e}=M(G \underset{\alpha}{\times} \mathscr{F})$ because a nonzero state on a two sided ideal of a $C^{*}$-algebra extends uniquely to a state on that $C^{*}$-algebra. Next we need to check that $\tilde{\omega} \mid \mathscr{F}=\omega$. By the uniqueness of the extension, it suffices to check its properties on $L^{1}(G, \mathscr{F})$. $\mathscr{F}$ acts on $L^{1}(G, \mathscr{F})$ by $f(r) \rightarrow A f(r), A \in \mathscr{F}$, and $\tilde{\omega}(A f)=$ $\omega\left(A \int f(r) \Delta(r)^{-1 / 2} d \lambda(r)\right)$. However $L^{1}(G, \mathscr{F})$ has an approximate identity $\left\{f_{\alpha}\right\}$, and hence $\tilde{\omega}\left(A f_{\alpha}\right) \rightarrow \omega(A) \forall A \in \mathscr{F}$. So $\tilde{\omega} \mid \mathscr{F}=\omega$. Clearly, since non $G$-invariant states can also be extended to $\mathscr{F}_{e}$, not all states of $\mathscr{F}_{e}$ can be obtained in this way. Finally we need to verify that $\tilde{\omega} \in \wp_{D}\left(\mathscr{F}_{e}\right)$, i.e. that $\tilde{\omega}(U(t))=1 \forall t \in G$. Again, by uniqueness of extension, this property is only checked on $L^{1}(G, \mathscr{F})$.

$$
\begin{aligned}
\tilde{\omega}(U(t) f) & =\omega\left(\int \Delta(t)^{1 / 2} \alpha_{t}\left(f\left(t^{-1} r\right)\right) \Delta(r)^{-1 / 2} d \lambda(r)\right) \\
& =\omega\left(\int f\left(t^{-1} r\right) \Delta(t)^{1 / 2} \Delta(r)^{-1 / 2} d \lambda(r)\right) \\
& =\omega\left(\int f(r) \Delta(r)^{-1 / 2} d \lambda(r)\right)=\tilde{\omega}(f) \quad \forall f \in L^{1}(G, \mathscr{F}) .
\end{aligned}
$$

Hence $\tilde{\omega} \in \wp_{D}\left(\mathscr{F}_{e}\right)$.

The nontriviality requirement easily adapts to an internal criterion on $\mathscr{F}$.

Corollary 3.4. $1 \notin \mathscr{A}(L)$ iff $1 \notin \mathscr{D}_{e} \cap \mathscr{F}$ iff $\wp^{G}(\mathscr{F}) \neq \varnothing$.

Proof. Since $\mathbf{1} \in \mathscr{F}$, we have by Theorems 2.4 and 3.3 that $\mathbf{1} \in \mathscr{A}(L)$ $\subset \mathscr{D}_{e}$ iff $\mathbf{1} \in \mathscr{D}_{e} \cap \mathscr{F} \subset \operatorname{Ker} \omega \forall \omega \in \wp^{G}(\mathscr{F})$.

Next we examine the various state spaces. Since $\wp^{G}(\mathscr{F})$ is a convex $w^{*}$-closed compact set, the Krein-Milman theorem applies.

Theorem 3.5. (i) We have the following surjections:

$$
\wp^{G}(\mathscr{F}) \stackrel{r}{\longrightarrow} \wp_{\mathscr{P}}\left(\mathcal{O}_{e} \cap \mathscr{F}\right) \stackrel{f}{\longrightarrow} \wp(\mathscr{R}),
$$

where $r: \mathscr{F}^{*} \mapsto\left(\mathcal{O}_{e} \cap \mathscr{F}\right)^{*}$ is restriction of functionals,

$$
\wp_{\mathscr{D}}\left(\mathcal{O}_{e} \cap \mathscr{F}\right):=\left\{\omega \in \wp\left(\mathcal{O}_{e} \cap \mathscr{F}\right) \mid \mathscr{D} \in \operatorname{Ker} \omega\right\}
$$

and $f: \wp_{\mathscr{D}}\left(\mathcal{O}_{e} \cap \mathscr{F}\right) \mapsto \wp(\mathscr{R})$, the canonical map, is a $w^{*}$-continuous isometric bijection.

(ii) There is a bijection between $\wp_{\mathscr{R}}^{p}\left(\mathcal{O}_{e} \cap \mathscr{F}\right)$ and $\wp^{p}(\mathscr{R})$.

Proof. (i) Clearly by 3.3 , if $\omega \in \wp^{G}(\mathscr{F})$, then $\omega \mid\left(\mathcal{O}_{e} \cap \mathscr{F}\right)$ vanishes on $\mathscr{D}$. Conversely, for an $\omega \in \wp_{\mathscr{D}}\left(\mathcal{O}_{e} \cap \mathscr{F}\right)$ we have by construction that on extension to a Dirac state on $\mathscr{F}_{e}$, there are no contradictions. On using 3.3 again, we see that $r$ is a surjection. That $f$ is an isometric $w^{*}$-continuous bijection, follows from Dixmier 2.11.6 [10].

(ii) Apply Dixmier 2.11.8.

In the case of inner constraints, the statement of 2.10 expresses the connection with 
the heuristic theory, and in this situation the similar statement follows almost trivially. First write the condition $\pi_{\omega}(\mathscr{D}) \Omega_{\omega}=0$ as $\pi_{\omega}(U) \Omega_{\omega}=\Omega_{\omega} \forall U \in \mathscr{U}$, and return to the outer constraint situation. Given an $\omega \in \wp^{G}(\mathscr{F})$, we have a covariant representation $\left(\pi_{\omega}, U^{\omega}, \mathscr{H}_{\omega}, \Omega_{\omega}\right)$ within which $U_{G}^{\omega} \Omega_{\omega}=\Omega_{\omega}$. Now by Pedersen 7.6.6 [8], for each covariant representation of $\mathscr{F}$, there is a unique nondegenerate representation of $\mathscr{F}_{e}$ over the same representation space. Hence denote this representation of $\mathscr{F}_{e}$ associated to $\left(\pi_{\omega}, U^{\omega}\right)$ by $\rho_{\omega}$. Then by Pedersen 7.6.4, $U_{g}^{\omega}=\rho_{\omega}\left(U_{g}\right) \forall g \in G$ and $\pi_{\omega}(A)=\rho_{\omega}(A) \forall A \in \mathscr{F}$. Then $\rho_{\omega}\left(U_{G}\right) \Omega_{\omega}=\Omega_{\omega}$. Conversely, if $\rho_{\omega}\left(U_{G}\right) \Omega_{\omega}=\Omega_{\omega}$, then $\tilde{\omega}(\cdot):=\left(\Omega_{\omega}, \rho_{\omega}(\cdot) \Omega_{\omega}\right)$ is a Dirac state on $\mathscr{F}_{e}$, and restricted to $\mathscr{F}$ is in $\wp^{G}(\mathscr{F})$.

Next we need to specify the automorphisms on $\mathscr{F}$ which are compatible with the present construction. Let the set of all possibly relevant automorphisms on $\mathscr{F}$ comprise a locally compact group $H$ which contains the gauge group $G$. Retain the previous notation for the action $\alpha: H \supset G \mapsto$ Aut. $\mathscr{F}$. Since the Haar measure of $H$ may not restrict to the Haar measure of $G$, it is not in general true that $G \times \underset{\alpha}{\times} \mathscr{F}$ $\subset H \underset{\alpha}{\times} \mathscr{F}$. First a general theorem.

Theorem 3.6. For the situation $\alpha: H \supset G \mapsto$ Aut $\mathscr{F}$, we have identifications such that:

(i) $G \underset{\alpha}{\times} \mathscr{F} \subset M(H \underset{\alpha}{\times} \mathscr{F})$,

(ii) $\mathscr{F} \cup U(G) \subset M(G \times \underset{\alpha}{\mathscr{F}}) \cap M(H \underset{\alpha}{\times} \mathscr{F})$,

(iii) $C^{*}(\mathscr{F} \cup U(G)) \subset C^{*}(\mathscr{F} \cup U(H))$.

Proof. (i) Define a homomorphism $L: G \underset{\alpha}{\times} \mathscr{F} \mapsto M(H \underset{\alpha}{\times} \mathscr{F})$ by:

$$
(L(f) k)(h):=\int_{G} f(g) \alpha_{g}\left(k\left(g^{-1} h\right)\right) d \lambda(g),
$$

where $f \in L^{1}(G, \mathscr{F}), k \in L^{1}(H, \mathscr{F})$, and the right-hand side defines an element of $L^{1}(H, \mathscr{F})$. Then $L$ can be taken as an identification.

(ii) We need to show that $\pi(\mathscr{F})$ and $U(G)$ (defined as operators on $L^{1}(H, \mathscr{F})$ ), act in the same way on the subspace $L^{1}(G, \mathscr{F}) \subset L^{1}(H, \mathscr{F})$ as the corresponding operators defined on $L^{1}(G, \mathscr{F})$ :

$$
\begin{aligned}
\pi(A)(L(f) k)(h) & =A(L(f) k)(h)=\int_{G} A f(g) \alpha_{g}\left(k\left(g^{-1} h\right)\right) d \lambda(g) \\
& =(L(\pi(A) f) k)(h)
\end{aligned}
$$

$\forall f \in L^{1}(G, \mathscr{F}), k \in L^{1}(H, \mathscr{F})$. Then with the same notation and with $s \in G$ :

$$
\begin{aligned}
U(s)(L(f) k)(h) & =U(s) \int_{G} f(g) \alpha_{g}\left(k\left(g^{-1} h\right)\right) d \lambda(g) \\
& =\int_{G} \Delta(s)^{1 / 2} \alpha_{s}\left(f(g) \alpha_{g}\left(k\left(g^{-1} s^{-1} h\right)\right)\right) d \lambda(g) \\
& =\int_{G} \Delta(s)^{1 / 2} \alpha_{s}(f(g)) \alpha_{s g}\left(k\left(g^{-1} s^{-1} h\right)\right) d \lambda(g) \\
& =\int_{G} \Delta(s)^{1 / 2} \alpha_{s}\left(f\left(s^{-1} g\right)\right) \alpha_{g}\left(k\left(g^{-1} h\right)\right) d \lambda(g) \\
& =(L(U(s) f) k)(h),
\end{aligned}
$$

and hence we can say $\mathscr{F} \cup U(G) \subset M(G \underset{\alpha}{\times} \mathscr{F}) \cap M(H \underset{\alpha}{\times} \mathscr{F})$. 
(iii) We already have that $C^{*}(\mathscr{F} \cup U(G)) \subset M(G \underset{\alpha}{\times} \mathscr{F})$ and also $C^{*}(\mathscr{F} \cup U(H))$ $\subset M(H \underset{\alpha}{\times} \mathscr{F})$, and hence by (ii), we only need to show that the $C^{*}$-norm of $M(G \underset{\alpha}{\times} \mathscr{F})$ is equal to the $C^{*}$-norm of $M(H \underset{\alpha}{\times} \mathscr{F})$ on their intersection. However we already have that $G \times \underset{\alpha}{\times} \mathscr{F} \subset M(H \underset{\alpha}{\times} \mathscr{F})$, and the $C^{*}$-norm of $M(G \underset{\alpha}{\times} \mathscr{F})$ derives from the norm of $G \times \underset{\alpha}{\times} \mathscr{F}$. Hence the $C^{*}$-norms are equal.

Remark. From the last part of the proof of the theorem, we see that $C^{*}(\mathscr{F} \cup U(G))$ is the same in both $M(G \underset{\alpha}{\times} \mathscr{F})$ and $M(H \underset{\alpha}{\times} \mathscr{F})$, and so we can say that

$$
C^{*}(\mathscr{F} \cup U(G)) \subset M(G \underset{\alpha}{\times} \mathscr{F}) \cap M(H \underset{\alpha}{\times} \mathscr{F}) .
$$

Each $h \in H$ defines an automorphism Ad $U(h)$ which restricts to $\alpha_{h}$ on $\mathscr{F}$, and so we are led to ask, can this automorphism be extended to $\mathscr{F}_{e}=M\left(G \times{ }_{\alpha} \mathscr{F}\right) \forall h$ ? When $h \in H \backslash G$, it is not clear that this should be so, because in $(U(h) f)(r):=$ $\Delta(h)^{1 / 2} \alpha_{h}\left(f\left(h^{-1} r\right)\right)$, the support of $f \in L^{1}(G, \mathscr{F})$ has been shifted by $h^{-1}$, and so $U(h) f \notin L^{1}(G, \mathscr{F})$. More precisely, for $(f, k) \in L^{1}(G, \mathscr{F}) \times L^{1}(H, \mathscr{F})$, and $h \in H \backslash G$ :

$$
\begin{aligned}
((\operatorname{Ad} U(h) \circ L(f)) k)(r) & =U(h)^{-1} L(f) U(h) k(r) \\
& =U\left(h^{-1}\right) L(f) \Delta(h)^{1 / 2} \alpha_{h}\left(k\left(h^{-1} r\right)\right) \\
& =U\left(h^{-1}\right) \int_{G} f(g) \Delta(h)^{1 / 2} \alpha_{g h}\left(k\left(h^{-1} g^{-1} r\right)\right) d \lambda(g) \\
& =\int_{G} \alpha_{h^{-1}}(f(g)) \alpha_{h^{-1} g h}\left(k\left(h^{-1} g^{-1} h r\right)\right) d \lambda(g) .
\end{aligned}
$$

This can be written in the form $L(t) k$ with $t \in L^{1}(G, \mathscr{F})$, only if $h^{-1} G h \subset G$. Naturally, that would be the case when $G$ is a normal subgroup of $H$, but that excludes most physical situations, since $G$ is a gauge group. So $\alpha_{h}$ can be extended to $\mathscr{F}_{e}$ only if $h \in \operatorname{St}(G):=\left\{h \in H \mid h^{-1} G h \subseteq G\right\}$. This indicates that $\mathscr{F}_{e}$ may be inconvenient as a universe to work in. Now by Theorem 3.2, the algebras $\mathcal{O}_{e} \cap \mathscr{F}$ and $\mathscr{D}_{e} \cap \mathscr{F}$ in $\mathscr{F}$ would be unaffected by the choice of $\mathscr{F}_{e}$, as long as it contains $U(G) \cup \mathscr{F}$. Now since $\mathscr{F} \cup U(G) \subset M(G \underset{\alpha}{\times} \mathscr{F}) \cap M(H \underset{\alpha}{\times} \mathscr{F})$, we have $C^{*}(U(G) \cup \mathscr{F}) \subset M(H \underset{\alpha}{\times} \mathscr{F})$, and hence we could just as well have chosen $\mathscr{F}_{e^{\prime}}:=M(H \underset{\alpha}{\times} \mathscr{F})$ for carrying the $T$ procedure on $U(G)$ out in, instead of $\mathscr{F}_{e}$. Henceforth this will be the course that we will follow, and we omit the prime on the subscript ' $e$ '.

The set of physically admissible automorphisms analogous to the case of inner constraints, will consist of all the automorphisms definable on the final physical algebra $\mathscr{R}_{c}:=\mathcal{O}_{c} / \mathscr{D}_{c}$, where on the basis of physical arguments one would have chosen $\mathscr{O}_{c} \subset \mathcal{O}_{e} \cap \mathscr{F}$ and $\mathscr{D}_{c}:=\mathscr{D}_{e} \cap \mathcal{O}_{c}$. $\mathscr{R}_{c}$ must be simple. As before, we are also interested in the set of automorphisms of $\mathscr{F}$ which are definable on the maximal physical algebra $\mathscr{R}$ :

$$
\mathbf{r}:=\left\{\beta \in \text { Aut } \mathscr{F} \mid \beta\left(\mathcal{O}_{e} \cap \mathscr{F}\right) \subseteq \mathcal{O}_{e} \cap \mathscr{F}, \quad \beta\left(\mathscr{D}_{e} \cap \mathscr{F}\right) \subseteq \mathscr{D}_{e} \cap \mathscr{F}\right\}
$$

Then clearly $h \in \operatorname{St}(G) \Rightarrow \alpha_{h} \in \mathbf{Y}$, and also $\alpha_{h} \in \mathbf{\Upsilon}$ if $\alpha_{h}\left(\mathscr{D}_{e}\right) \subseteq \mathscr{D}_{e}$.

Theorem 3.7. $\alpha_{h}\left(\mathscr{D}_{e}\right) \subseteq \mathscr{D}_{e}$ iff $\omega\left(U\left(h^{-1} G h\right)\right)=1 \forall \omega \in \wp_{D e}$.

Proof. Let $\alpha_{h}\left(\mathscr{D}_{e}\right)=\operatorname{Ad} U(h) \circ \mathscr{D}_{e} \subset \mathscr{D}_{e}$. Then since $L(G):=U(G)-\mathbf{1} \subset \mathscr{D}_{e}$, we see $\operatorname{Ad} U(h) \circ L(g)=\operatorname{Ad} U(h) \circ(U(g)-\mathbf{1})=U\left(h^{-1} g h\right)-\mathbf{1} \in \mathscr{D}_{e} \subset \operatorname{Ker} \omega \forall \omega \in \wp_{D e}$, hence $\omega\left(U\left(h^{-1} g h\right)\right)=1 \forall \omega \in \wp_{D e}, g \in G$. 
Conversely, if $\omega\left(U\left(h^{-1} g h\right)\right)=1 \forall \omega \in \wp_{D e}$, then $\alpha_{h}(\mathscr{A}(L)) \subset \mathscr{D}_{e}$.

At this point we have collected enough general structure to deal with an example.

\section{Structure of the Heuristic Gupta-Bleuler Theory}

We record here the heuristic Gupta-Bleuler structure for purposes of comparison with the ensuing rigorous reconstruction $[11,12]$. The Lagrangian is

$$
L=-\frac{1}{4} \int d^{4} x F_{\mu \nu} F^{\mu \nu}, \text { where } F_{\mu \nu}(x):=A_{v, \mu}(x)-A_{\mu, \nu}(x) .
$$

Then the field equation is $F_{\mu \nu}{ }^{, v}(x)=0$ and the CCR's are

$$
\left[A_{\mu}(x), A_{v}\left(x^{\prime}\right)\right]=-i g_{\mu \nu} D\left(x-x^{\prime}\right), \quad D(x):=-(2 \pi)^{-3} \int_{C_{+}} \frac{d^{3} k}{k_{0}} e^{i k \cdot x} \sin k_{0} x_{0},
$$

and $C_{+}$is the mantle of the positive light-cone, $k_{0}=|\underline{k}|$. The Fourier transform is written:

$$
A_{\mu}(x)=\left(2(2 \pi)^{3}\right)^{-1 / 2} \int_{C_{+}} \frac{d^{3} k}{k_{0}}\left(a_{\mu}(\underline{k}) e^{-i k \cdot x}+a_{\mu}^{*}(\underline{k}) e^{i k \cdot x}\right)
$$

with CCR's: $\left[a_{\mu}(\underline{k}), a_{v}^{*}\left(\underline{k}^{\prime}\right)\right]=-g_{\mu v} k_{0} \delta^{3}\left(\underline{k}-\underline{k}^{\prime}\right) \mid C_{+}$and the other commutators involving $a$ are zero. At this point $A_{\mu}(x)$ does not yet satisfy the field equations. On smearing we obtain:

$$
A(F):=\int d^{4} x A_{\mu}(x) f^{\mu}(x)=\sqrt{\pi} \int_{C_{+}} \frac{d^{3} k}{k_{0}}\left(a_{\mu}(\underline{k}) \hat{f}^{\mu}(k)+a_{\mu}^{*}(\underline{k}) \overline{\left.\hat{f}^{\mu}(k)\right)},\right.
$$

where

$$
F=\left(f^{0}, f^{1}, f^{2}, f^{3}\right) \in \mathscr{S}^{4}\left(\mathbb{R}^{4}\right)
$$

and

$$
\begin{aligned}
\hat{f}_{\mu}(k) & :=(2 \pi)^{-2} \int d^{4} x e^{-i k \cdot x} f_{\mu}(x) \in \mathscr{S}_{r}^{4}\left(\mathbb{R}^{4}\right), \\
\mathscr{S}_{r}^{4}\left(\mathbb{R}^{4}\right) & :=\left\{F \in \mathscr{S}_{C}^{4}\left(\mathbb{R}^{4}\right) \mid \bar{F}(k)=F(-k)\right\} .
\end{aligned}
$$

The symbol $\mathscr{S}(\mathbb{R})$ denotes Schwartz space on $\mathbb{R}$. The operators $a_{\mu}(\underline{k}), a_{\mu}^{*}(\underline{k})$ and hence $A(F)$ act on the space $\mathscr{H}$ defined below. Equip $\mathscr{S}_{r}^{4}\left(\mathbb{R}^{4}\right)$ with the scalar product $\langle f \mid h\rangle^{(1)}:=\int_{C_{+}}\left(d^{3} k / k_{0}\right) \sum_{\mu=0}^{3} \bar{f}_{\mu}(k) h_{\mu}(k)$, then factor out the zero norm part, and complete it to obtain the Hilbert space $\mathscr{H}^{(1)}$. An indefinite sesquilinear form is given on $\mathscr{H}^{(1)}$ by

$$
(f, h)^{(1)}:=-\langle f \mid g h\rangle^{(1)}=-\int_{C_{+}} \frac{d^{3} k}{k_{0}} \bar{f}_{\mu}(k) h^{\mu}(k) .
$$

Then $\mathscr{H}:=\mathbf{C} \oplus\left(\bigoplus_{n=1}^{\infty}\left(\mathscr{H}^{(1) \otimes n}\right)_{s}\right)$, where the subscript 's' means symmetric tensor product. $\mathscr{H}$ is a Hilbert space with the scalar product $\langle\cdot \mid \cdot\rangle$ obtained from $\langle\cdot \mid \cdot\rangle^{(1)}$, and is a Krein space with the indefinite form $(\cdot, \cdot)$ obtained from $(\cdot, \cdot)^{(1)}$, and $a_{\mu}$ and $a_{\mu}^{*}$ act in the usual way as annihilation and creation operators on $\mathscr{H}$. 
A supplementary condition

$$
\chi(x):=\partial^{\mu} A_{\mu}^{(+)}(x)=-i\left(2(2 \pi)^{3}\right)^{-1 / 2} \int_{C_{+}} \frac{d^{3} k}{k_{0}} k^{\mu} a_{\mu}(k) e^{-i k \cdot x}
$$

selects the physical subspace $\mathscr{H}^{\prime}:=\{\Phi \in \mathscr{H} \mid \chi(x) \Phi=0\}$, and on $\mathscr{H}^{(1)}$, this is exactly the space of functions satisfying $k^{\mu} f_{\mu}(k)=0$, denoted $\mathscr{H}^{(1)^{\prime}}$, and moreover, $(\cdot, \cdot)$ is positive on $\mathscr{H}^{\prime}$. Now $(\cdot, \cdot)$ is preferable to $\langle\cdot \mid \cdot\rangle$, because if the Poincare transformations are defined in the natural way: $(\Lambda, a) F(k)=e^{i a \cdot k} \Lambda F\left(\Lambda^{-1} k\right)$, we note that $(\cdot, \cdot)$ is invariant under these but $\langle\cdot \mid \cdot\rangle$ is not. So by factoring out $\mathscr{H}^{\prime \prime}:=\operatorname{Ker}(\cdot, \cdot) \cap \mathscr{H}^{\prime}$, we can make a Hilbert space $\mathscr{H}_{\text {phys }}:=\mathscr{H}^{\prime} / \mathscr{H}^{\prime \prime}$, which carries a unitary representation of the orthochronous Poincaré group. This factoring is justified through imposition of the field equations cf. [12]: In $\mathscr{H}^{(1)}$, the space $\mathscr{H}^{(1)^{\prime \prime}}$ consists of all the gradients, i.e. $f_{\mu}(k)=k_{\mu} f(k)$. After smearing with $F_{\mu}(k)=\left(k_{\mu} k^{v}-k^{2} \delta_{\mu \nu}\right) f_{\nu}(k), A(F)$ will correspond to $F_{\mu \nu}{ }^{, v}(f)$, and since $\mathscr{H}^{(1)}$ is defined on $C_{+}$, the set of test functions $\left\{k_{\mu} k^{v} f_{v}(k)\left|C_{+}\right| f_{v} \in \mathscr{S}_{r}\left(\mathbb{R}^{4}\right)\right\}$ will represent the field equations. This is exactly $\mathscr{H}^{(1)^{\prime \prime}}$. Hence $F_{\mu \nu}{ }^{, v}(f) \mathscr{H}^{\prime} \subset \mathscr{H}^{\prime \prime}$, and so in the representation canonically defined on $\mathscr{H}_{\text {phys }}$, $F_{\mu \nu}{ }^{, \nu}(f)$ will be zero, and the field equations are now satisfied. Note that the field equations are imposed as state conditions. On smearing $F_{\mu \nu}$ with an antisymmetric tensor function $f_{\mu v}$ to obtain $F(f)$, we note that the latter corresponds to the smearing of $A_{\mu}$ with $2 k_{v} f^{\mu v}$. Now clearly $\left(2 k_{v} f^{\mu \nu} \mid C_{+}\right) \in \mathscr{H}^{(1)^{\prime}}$, and so $F_{\mu v} \mathscr{H}^{(1)^{\prime}}$ $\subset \mathscr{H}^{(1)^{\prime}}$, i.e. $F_{\mu \nu}$ is representable on $\mathscr{H}_{\text {phys }}$.

To model this in rigorous field theory, we will proceed as follows. For the canonical structure, we take $\mathscr{F}=\overline{\Delta\left(\mathscr{H}^{(1)}, B\right)}$ cf. [7] with $B$ the appropriate symplectic form. For imposing the constraint $\chi(f)$, we need to calculate

$$
\left(\operatorname{Ad} \exp i \lambda \chi^{*}(f) \chi(f)\right) e^{i A(G)}=e^{i A(M)},
$$

and since this transformation $G \mapsto M$ in $\mathscr{H}^{(1)}$ is symplectic, it defines an automorphism on $\mathscr{F}$. Using the general theory of the previous section, we obtain a $C^{*}$-algebra $\mathscr{F}_{e} \supset \mathscr{F}$ containing unitaries $U$ which will implement the automorphisms above, and these are identified with the heuristic objects $\exp i \lambda \chi^{*}(f) \chi(f)$. The physical states of $\mathscr{F}$ are the covariant states with respect to these transformations, and the physical algebra is obtained as $C^{*}\left(\delta_{\mathscr{H}^{(1)}} \cap \mathcal{O}_{e}\right)$ and we find that it is exactly generated by $\mathscr{H}^{(1)^{\prime}}$, i.e. it is $C^{*}\left(\delta_{\mathscr{H}^{(1)^{\prime}}}\right)$. On imposing the field equation as a constraint, we obtain

$$
\mathscr{R}_{c}=C^{*}\left(\delta_{\mathscr{H}^{(1)^{\prime}}}\right) / C^{*}\left(\delta_{\mathscr{H}^{(1)^{\prime \prime}}}-\mathbf{1}\right) C^{*}\left(\delta_{\mathscr{P}^{(1)^{\prime}}}\right)
$$

and consequently show that $B$ is nondegenerate when defined on $\mathscr{H}^{(1)^{\prime}} / \mathscr{H}^{(1)^{\prime \prime}}=$ $\mathscr{H}_{\text {phys }}^{(1)}$, and indeed that $\mathscr{R}_{c}=\overline{\Delta\left(\mathscr{H}_{\text {phys }}^{(1)}, B\right)}$, which proves that the chosen physical algebra is simple. This is the abstract algebraic structure. To complete the parallel with the heuristic theory, we demonstrate that from the Fock-type generating functional $\omega\left(\delta_{F}\right)=\exp -\frac{1}{4}(F, F)^{(1)}$ we can construct a functional on $\mathscr{F}$ such that its (indefinite) GNS-construction has precisely the right structure. That is, the GNSrepresentation space has a Poincaré invariant indefinite inner product (IIP), the subspace generated by the action of $C^{*}\left(\delta_{\mathscr{H}}^{(1)^{\prime}}\right)$ on the cyclic vector is positive with respect to the IIP, and its null-space is exactly the space generated by $C^{*}\left(\delta_{\mathscr{H}^{(1)^{\prime \prime}}}-1\right) C^{*}\left(\delta_{\mathscr{H}^{(1)^{\prime}}}\right)$. These algebras also contain the right abstract objects corresponding to the heuristic ones. 


\section{Gupta-Bleuler Electromagnetism as an Algebraic Field Theory}

To prepare the ground for the abstract theory, it is necessary to calculate in the heuristic framework the object

$$
\left(\operatorname{Ad} \exp i \lambda \chi^{*}(f) \chi(f)\right)\left(e^{i A(G)}\right)
$$

On smearing, we obtain:

$$
\begin{aligned}
\Omega(f):=\chi^{*}(f) \chi(f) & =\int d^{4} x d^{4} x^{\prime} \chi^{*}(x) \chi\left(x^{\prime}\right) f(x) f\left(x^{\prime}\right) \\
& =\pi \int_{C_{+} C_{+}^{\prime}} \frac{d^{3} k}{k_{0}} \frac{d^{3} k^{\prime}}{k_{0}^{\prime}} k^{\mu} k_{\nu}^{\prime} a_{\mu}^{*}(\underline{k}) a^{v}(\underline{k}) \overline{\hat{f}}(k) \hat{f}\left(k^{\prime}\right) .
\end{aligned}
$$

Commutators:

$$
[A(G), \Omega(f)]=\sqrt{\pi} \int_{C_{+}} \frac{d^{3} k}{k_{0}}\left(a_{\mu}(\underline{k}) h^{\mu}(k)-a_{\mu}^{*}(\underline{k}) \bar{h}^{\mu}(k)\right),
$$

where

$$
\begin{aligned}
h^{\mu}(k) & :=-\pi k^{\mu} \hat{f}(k) \int_{C_{+}^{\prime}} \frac{d^{3} k^{\prime}}{k_{0}^{\prime}} g^{v}\left(k^{\prime}\right) k_{v}^{\prime} \overline{\hat{f}\left(k^{\prime}\right)} \\
{[A(G),[A(G), \Omega(f)]] } & =\pi \int_{C_{+}} \frac{d^{3} k}{k_{0}}\left(\hat{g}_{\mu}(k) \bar{h}^{\mu}(k)+\overline{\hat{g}}_{\mu}(k) h^{\mu}(k)\right) \in \mathbb{R}, \\
{[\Omega(f),[\Omega(f), A(G)]] } & =0,
\end{aligned}
$$

and so all higher commutators vanish. Using the Baker-Campbell-Hausdorff formula, we find $e^{-Y} e^{X} e^{Y}=\exp (X+[X, Y])$ if $[X,[X, Y]] \in \mathbb{R}$ and $[Y,[Y, X]]=0$. Hence

$$
\begin{aligned}
(\operatorname{Ad} \exp i \lambda \Omega(f))\left(e^{i A(G)}\right)= & \exp i \sqrt{\pi} \int_{C_{+}} \frac{d^{3} k}{k_{0}}\left\{a_{\mu}(\underline{k})\left(\hat{g}^{\mu}(k)+i \lambda h^{\mu}(k)\right)\right. \\
& \left.\quad+a_{\mu}^{*}(\underline{k}) \overline{\left(\hat{g}^{\mu}(k)\right.}-i \hat{\lambda} \overline{\left.h^{\mu}(k)\right)}\right\} \\
= & : e^{i A(M)},
\end{aligned}
$$

where $M$ is a function such that its Fourier transform satisfies:

$$
\hat{M}^{\mu}(k)=(2 \pi)^{-2} \int d^{4} x e^{-i k \cdot x} M^{\mu}(x)=\hat{g}^{\mu}(k)+i \lambda h^{\mu}(k) .
$$

For this to be acceptable in the present framework, we need to check that $M \in \mathscr{S}^{4}\left(\mathbb{R}^{4}\right)$, i.e. that $\hat{M}$ satisfies the reality condition: $\bar{m}(k)=m(-k)$. This is seen to hold from $\hat{g}_{\mu}(k) \in \mathscr{P}_{r}^{4}\left(\mathbb{R}^{4}\right)$, and $\bar{h}^{\mu}(k)=-h^{\mu}(-k)$. Hence the gauge transformation $\operatorname{Ad} \exp i \lambda \Omega(f)$ is well-defined, and consists of the addition of a divergence with the form of $h$. Explicitly, for each $f \in \mathscr{P}\left(\mathbb{R}^{4}\right)$, we have a gauge transformation on test function space $\mathscr{S}_{r}^{4}\left(\mathbb{R}^{4}\right)$, expressed by:

$$
\hat{g}_{\mu}(k) \rightarrow \hat{g}_{\mu}(k)+i \lambda h_{\mu}(k)=: \hat{g}_{\mu}(k)+\lambda\left(\mathbf{G}_{f} \hat{g}\right)_{\mu}(k) \equiv\left(\mathbf{T}_{f}^{\lambda} \hat{g}\right)_{\mu}(k)
$$

with

$$
\left(\mathbf{G}_{f} \hat{g}\right)_{\mu}(k):=-i \pi k^{\mu} \hat{f}(k) \int_{C_{+}} \frac{d^{3} k^{\prime}}{k_{0}^{\prime}} \hat{g}^{v}\left(k^{\prime}\right) k_{v}^{\prime} \overline{\hat{f}\left(k^{\prime}\right)} .
$$


Now in setting up the abstract theory, we take as our symplectic space $\mathscr{M}=\mathscr{H}^{(1)}$, i.e. factor out the zero-norm part of the scalar product $\langle f \mid g\rangle^{(1)}:=$ $\int_{C_{+}}\left(d^{3} k / k_{0}\right) \sum_{\mu=0}^{3} \bar{f}_{\mu} h_{\mu}$ and complete it. This disposes of the off $C_{+}$-parts. The symplectic form $B$ defined on $\mathscr{M}$ which will correspond to the CCR's is:

$$
B(f, g):=\int_{C_{+}} \frac{d^{3} k}{k_{0}}\left(f^{\mu}(k) \bar{g}_{\mu}(k)-\bar{f}^{\mu}(k) g_{\mu}(k)\right),
$$

and the linear field algebra is chosen as $\mathscr{F}=\overline{\Delta(\mathscr{M}, B)}$ cf. 2.13. The gauge transformations $\mathbf{T}_{f}^{\lambda}$ are well-defined on $\mathscr{M}$. The following useful facts are verified by substitution:

(i) $B\left(\mathbf{G}_{f} g, h\right)=-B\left(g, \mathbf{G}_{f} h\right)$,

(ii) $\mathbf{G}_{f} \mathbf{G}_{h} g=0$. Combining these:

(iii) $B\left(\mathbf{G}_{f} g, \mathbf{G}_{m} h\right)=0$, and on application of these:

$$
B\left(\mathbf{T}_{f}^{\lambda} g, \mathbf{T}_{f}^{\lambda} h\right)=B\left(g+\lambda \mathbf{G}_{f} g, h+\lambda \mathbf{G}_{f} h\right)=B(g, h),
$$

i.e. $\mathbf{T}_{f}^{\lambda}$ is symplectic, and so every $\mathbf{T}_{f}^{\lambda}$ will define an automorphism on $\mathscr{F}$ by:

$$
\alpha_{\lambda}^{f}\left(\delta_{g}\right):=\delta_{\mathrm{T}_{f}^{\prime}}=\delta_{g+\lambda \mathbf{G}_{f} g} .
$$

Furthermore, by (ii), $\mathbf{T}_{f}^{\lambda} \mathbf{T}_{h}^{\gamma} g=g+\lambda \mathbf{G}_{f} g+\gamma \mathbf{G}_{h} g$, and from this we see that the gauge transformations commute: $\left[\mathbf{T}_{f}^{\lambda}, \mathbf{T}_{h}^{\gamma}\right]=l$, and that there is a one parameter group defined for each $f \in \mathscr{S}\left(\mathbb{R}^{4}\right)$ by $\mathbf{T}_{f}^{\lambda} \mathbf{T}_{f}^{\gamma}=\mathbf{T}_{f}^{\lambda+\gamma}$. Because $\mathbf{G}_{f}$ is nonlinear in $f$, we cannot extend the group property beyond this. It appears that a natural group to construct is

$$
G:=\bigoplus_{f \in \mathscr{S}\left(\mathbb{R}^{4}\right)} \mathbb{R}_{(f)}
$$

The cardinality of the continuous functions is the same as that of the real line. Group multiplication is simply vector addition, and the topology is any topology induced from the topologies of the $\mathbb{R}$ 's. The group action on $\mathscr{F}$ is defined by

$$
\alpha_{g}:=\prod_{f \in \mathscr{S}\left(\mathbb{R}^{4}\right)} \alpha_{\lambda(f)}^{f},
$$

where $g \in G$ was expressed as a function $\lambda: \mathscr{S}\left(\mathbb{R}^{4}\right) \mapsto \mathbb{R}$ with finite support. Hence the product is finite. However, $G$ is not locally compact, and so it is not possible to construct $G \times \mathscr{F}$ as prescribed by the general theory. To alleviate this difficulty, we proceed as follows. Construct the directed set $I$ consisting of all finite subsets of $\mathscr{S}\left(\mathbb{R}^{4}\right)$, together with the partial ordering of set inclusion. Then for each $s \in I$, we can construct the finite Cartesian product $G_{s}:=\underset{f \in S}{\times} \mathbb{R}_{(f)}$, which of course is a locally compact subgroup of $G$, and it has the natural action of $G$ restricted to it, which we denote by $\alpha^{s}: G_{s} \mapsto$ Aut $\mathscr{F}$. Hence it is possible to define $\mathscr{F}_{(s)}:=G_{s} \times \alpha_{\alpha^{s}} \mathscr{F}_{\text {, }}$ and $\mathscr{F}_{e}^{(s)}:=M\left(\mathscr{F}_{(s)}\right)$. Then $\mathscr{F} \subset \mathscr{F}_{e}^{(s)}$, and $\alpha_{\lambda(f)}^{s}=\operatorname{Ad} U_{\lambda(f)}^{s}$ for each $\lambda(f) \in G_{s}, f \in s$, and also $U_{\lambda(f)}^{s} \in \mathscr{F}_{e}^{(s)}$. Now by Theorem 3.6(ii), for $\alpha: H \supset G \mapsto$ Aut $\mathscr{F}$ we have $\mathscr{F} \cup U(G)$ $\subset M(G \underset{\alpha}{\times} \mathscr{F}) \cap M(H \underset{\alpha}{x} \mathscr{F})$. Let $s, p \in I$, then we wish to check that there is an identification between $\mathscr{F} \subset \mathscr{F}_{e}^{(s)}$, and $\mathscr{F} \subset \mathscr{F}_{e}^{(p)}$, even though they act on different 
spaces. Since $s \cup p \in I$, we have identifications such that $\mathscr{F} \subset \mathscr{F}_{e}^{(s \cup p)} \cap \mathscr{F}_{e}^{(s)}$, $\mathscr{F} \subset \mathscr{F}_{e}^{(s \cup p)} \cap \mathscr{F}_{e}^{(p)}$, and hence $\mathscr{F}$ in $\mathscr{F}_{e}^{(s)}$ can be identified with $\mathscr{F}$ in $\mathscr{F}_{e}^{(p)}$ by transitivity. Furthermore, if $s, p \in I$ and $s \cap p \neq \emptyset$, we would like to identify $U_{\lambda(f)}^{s}$ with $U_{\lambda(f)}^{p}$ for all $f \in S \cap p$, in which case it is $U_{\lambda(f)}^{s \cap p}$. This identification follows directly from Theorem 3.6(ii). Henceforth omit the superscript on $U$. Now starting from $\mathscr{F}$ $\subset \bigcap\left\{\mathscr{F}_{e}^{(s)} \mid s \in I\right\}$ and $U_{G_{s}}, s \in I$, we can construct a family of $C^{*}$-algebras $\mathscr{A}^{(s)}:=C^{*}\left(\mathscr{F} \cup U_{G_{s}}\right)$ employing the $C^{*}$-norm of $\mathscr{F}_{e}^{(s)}$, and by Theorem 3.6(iii) we have that $\mathscr{A}^{(s)} \subseteq \mathscr{A}^{(p)}$ if $s \subseteq p$. Hence we have obtained a structure which satisfies Takeda's criterion cf. [13], and so there must exist a $C^{*}$-inductive limit

$$
\mathscr{F}_{e}:=\overline{\bigcup_{s \in I} \mathscr{A}^{(s)}}
$$

This will be the convenient universe $\mathscr{F}_{e}$ in which we choose to work, and it illustrates a method of generalising the locally compactness assumption of the general theory. Note that from Theorem 3.3, $\wp_{D e}(\mathscr{F}(s)) \mid \mathscr{F}=\wp^{G_{s}}(\mathscr{F})$ and hence on employing Theorem 3.2, $\wp_{D e}\left(\mathscr{F}_{e}\right)=\bigcap_{s \in I}\left(\wp_{D e}\left(\mathscr{F}_{e}^{(s)}\right) \vDash \mathscr{F}_{e}\right)$ where $\vDash$ means "extended to." Hence $\wp_{D e}(\mathscr{F} e) \mid \mathscr{F}=\bigcap_{s \in I} \wp^{G_{s}}(\mathscr{F})=\wp^{G}(\mathscr{F})$, and so, just as before, we can carry the $T$ procedure through in $\mathscr{F}_{e}$, and then restrict to $\mathscr{F}$ to obtain the algebraic structure that result from the selection of the gauge invariant states: $\mathscr{D}=\mathscr{D}_{e} \cap \mathscr{F}, \mathcal{O}=\mathcal{O}_{e} \cap \mathscr{F}$, $\mathscr{R}=\mathcal{O} / \mathscr{D}$. As for nontriviality, that will be demonstrated by obtaining a nontrivial result.

Since the set $\delta_{\mathscr{M}}$ generates $\mathscr{F}$, we start by examining its behaviour. The set $\wp^{G}(\mathscr{F})$ is characterised by $\left(\alpha_{G}-l\right) \mathscr{F} \subset \operatorname{Ker} \omega \forall \omega \in \wp^{\mathfrak{O}^{G}}(\mathscr{F})$. Recall that $\mathscr{D}$ is the largest $C^{*}$ algebra on which all $\wp^{G}(\mathscr{F})$ vanish. Moreover, as was remarked above Theorem 3.2, $A \in \mathcal{O}_{e} \cap \mathscr{F}$ iff $\left(\alpha_{G}-l\right) A \subset \mathscr{D}$, and so we examine the set $\delta_{\mathscr{M}} \cap \mathcal{O}_{e}$.

Lemma 5.1. For an $F \in \mathscr{M}, \delta_{F} \in \mathcal{O}_{e}$ iff $\omega\left(\delta_{\lambda_{\mathrm{G}} F}\right)=1 \forall f \in \mathscr{S}\left(\mathbb{R}^{4}\right), \lambda \in \mathbb{R}, \omega \in \wp^{G}(\mathscr{F})$, and in this case

$$
B\left(F, \mathbf{G}_{f} F\right)=0 \forall f \in \mathscr{S}\left(\mathbb{R}^{4}\right) .
$$

Proof. Let $\left(l-\alpha_{\lambda(f)}\right) \delta_{F} \in \mathscr{D}$ with $\lambda(f) \in G$. Denote $\mathbf{T}_{\lambda(f)} \equiv \mathbf{T}_{f}^{\lambda(f)}$. Now: $\left(l-\alpha_{\lambda(f)}\right) \delta_{F}=$ $\delta_{F}-\delta_{\mathrm{T}_{\lambda(f)} F}=\delta_{F}\left(\mathbf{1}-\delta_{\lambda \mathbf{G}_{f} F} \exp (i \lambda / 2) B\left(F, \mathbf{G}_{f} F\right)\right)=\left(\mathbf{1}-\delta_{\lambda \mathbf{G}_{f} F} \exp \left((-i \lambda / 2) B\left(F, \mathbf{G}_{f} F\right)\right)\right) \delta_{F}$. By general theory, $\mathscr{D}=\bigcap\left\{N_{\omega} \cap N_{\omega}^{*} \mid \omega \in \wp^{G}(\mathscr{F})\right\}$, and each $N_{\omega}$ is a left ideal of $\mathscr{F}$. Hence $\left(l-\alpha_{\lambda(f)}\right) \delta_{F} \in N_{\omega} \cap N_{\omega}^{*} \Rightarrow \mathbf{1}-\delta_{\lambda_{\mathbf{G}_{f} F}} \exp (i \lambda / 2) B\left(F, \mathbf{G}_{f} F\right) \in$ $\operatorname{Ker} \omega \ni \mathbf{1}-\delta_{i \mathbf{G}_{f} F} \exp (-i \lambda / 2) B\left(F, \mathbf{G}_{f} F\right)$. So $\quad 1=\omega\left(\delta_{\lambda \mathbf{G}_{f} F}\right) \exp (i \lambda / 2) B\left(F, \mathbf{G}_{f} F\right)=$ $\omega\left(\delta_{\lambda \mathbf{G}_{f} F}\right) \exp (-i \lambda / 2) B\left(F, \mathbf{G}_{f} F\right) \forall \lambda \in \mathbb{R}, f \in \mathscr{S}\left(\mathbb{R}^{4}\right)$, and hence $B\left(F, \mathbf{G}_{f} F\right)=0 \forall f$, and $\omega\left(\delta_{\lambda \mathbf{G}_{f} F}\right)=1 \forall \omega \in \wp^{G}(\mathscr{F}), \lambda \in \mathbb{R}$, and $f \in \mathscr{S}\left(\mathbb{R}^{4}\right)$. Conversely, let $\omega\left(\delta_{\lambda \mathbf{G}_{f} F}\right)=1 \forall \lambda, f$, then $\omega\left(\delta_{F} \delta_{i \mathbf{G}_{f} F}\right)=\omega\left(\delta_{\lambda \mathbf{G}_{f} F} \delta_{F}\right)=\omega\left(\delta_{F}\right)$ cf. statements following 2.2 , and so $\omega\left(\delta_{\mathrm{T}_{\lambda(f)} F}\right) \exp (i \lambda / 2) B\left(F, \mathbf{G}_{f} F\right)=\omega\left(\delta_{\mathrm{T}_{\lambda(f)} F}\right) \exp (-i \lambda / 2) B\left(F, \mathbf{G}_{f} F\right)=\omega\left(\delta_{F}\right) \forall \lambda, f$ and so $B\left(F, \mathbf{G}_{f} F\right)=0$, and $\omega\left(\delta_{\mathrm{T}_{\lambda(f)}}\right)=\omega\left(\delta_{F}\right)$, which we know already. Moreover, from $\omega\left(\delta_{\lambda \mathbf{G}_{f} F}\right)=1$ and $\left(\delta_{\mathrm{T}_{\lambda(f)} F}-\delta_{F}\right)^{*}\left(\delta_{\mathrm{T}_{\lambda(f)}}-\delta_{F}\right)=2-\delta_{\lambda \mathbf{G}_{f} F}-\delta_{-\lambda \mathbf{G}_{f} F}$ we see that $\left(\alpha_{\lambda(f)}-\imath\right) \delta_{F} \in N_{\omega}$, and in a similar way we can see that it is in $N_{\omega}^{*}$. Hence using $\mathscr{D}=\bigcap\left\{N_{\omega} \cap N_{\omega}^{*} \mid \omega \in \wp^{G}(\mathscr{F})\right\}$ the result follows that $\left(\alpha_{\lambda(f)}-l\right) \delta_{F} \in \mathscr{D}$. 
So $\delta_{F} \in \mathcal{O}_{e} \Rightarrow B\left(F, \mathbf{G}_{f} F\right)=0 \forall f \in \mathscr{S}\left(\mathbb{R}^{4}\right)$. We find by substitution that

$$
B\left(F, \mathbf{G}_{g} F\right)=i 2 \pi\left|\int_{C_{+}} \frac{d^{3} k}{k_{0}} k_{\mu} f^{\mu}(k) \hat{g}(k)\right|^{2}=0 \quad \forall g \in \mathscr{S}\left(\mathbb{R}^{4}\right),
$$

and hence $k_{\mu} f^{\mu}(k) \mid C_{+}=0$. This is exactly the subspace $\mathscr{H}^{(1)^{\prime}}$ selected by the supplementary condition in the heuristic situation. Henceforth denote it by $\mathscr{Q}$. Moreover, from the explicit form of $\mathbf{G}_{f}$, we find that $\mathbf{G}_{f} \mathscr{2}=0$, i.e. these are the gauge invariant elements. Now from 5.1, the fact that $\omega\left(\delta_{i \mathrm{G}_{f^{2}}}\right)=\omega(\mathbf{1})=1$ and the $T$ procedure will give the reverse implication, so that we can say that $\delta_{\mathscr{A}} \cap \mathcal{O}_{e}=\delta_{2}$. This is the analogue of the inner constraint situation for linear bosons, where we found that $\delta_{\mathscr{M}} \cap \mathcal{O}=\delta_{\mathbf{P}}=\delta_{\mathscr{M}} \cap \mathscr{A}(L)^{\prime}$ cf. Sect. 2. We choose as our physical algebra $\mathcal{O}_{c}=C^{*}\left(\delta_{2}\right)$, because it is difficult to get our hands on the additional elements in $\left(\mathcal{O}_{e} \cap \mathscr{F}\right) \backslash \mathcal{O}_{c}$. There may be for instance elements of the form $\sum_{i} \lambda_{i} \delta_{F_{i}} \in \mathcal{O}_{e} \cap \mathscr{F}$ such that $F_{i} \notin \mathscr{Q} \forall i$. Moreover, the fact that $\mathscr{Q}$ is the physical space justifies our choice.

The orthochronous Poincaré transformations (used to preserve $C_{+}$), are defined in the natural way on $\mathscr{M}:(\Lambda, a) F(k):=e^{\imath a \cdot k} \Lambda F\left(\Lambda^{-1} k\right)$, and as these are symplectic, will define automorphisms on $\mathscr{F}$. These automorphisms will preserve $\mathcal{O}_{c}$, because $\mathscr{Q}$ is invariant under $(\Lambda, a)$.

At this point, we note that $B$ is degenerate on $\mathscr{Q}$, and hence $\mathcal{O}_{c}$ is not simple, and also that we still have to impose the field equation as a constraint. In order to do that, we digress here to the more general situation of linear boson fields to prove two useful theorems.

Theorem 5.2. Given a test function space $H$ with a degenerate symplectic form $B$ on it, denote its degenerate part by $H_{0}$ and specify a constraint set $\mathscr{C} \subset H_{0}$. Then $B$ is naturally defined on $H / \mathscr{C}$ because $B\left(F+C_{1}, G+C_{2}\right)=B(F, G)=: \tilde{B}([F],[G])$ $\forall C_{i} \in \mathscr{C}$ and $F, G \in H$, where $[F] \in H / \mathscr{C}$ denotes the equivalence class of $F \in H$. Then:

$$
\overline{\Delta(H, B)} / C^{*}\left(\delta_{\mathscr{G}}-\mathbf{1}\right) \overline{\Delta(H, B)} \cong \overline{\Delta(H / \mathscr{C}, \widetilde{B})},
$$

where $C^{*}\left(\delta_{\mathscr{C}}-\mathbf{1}\right)$ is the $C^{*}$-algebra in $\overline{\Delta(H, B)}$ generated by $\left\{\delta_{C}-1 \mid C \in \mathscr{C}\right\}$. Since $\mathscr{C}$ $\subset H_{0}$, this latter $C^{*}$-algebra is in the centre of $\overline{\Delta(H, B)}$.

Proof. Let $\tau: \overline{\Delta(H, B)} \mapsto \overline{\Delta(H, B)} / C^{*}\left(\delta_{\mathscr{C}}-1\right) \overline{\Delta(H, B)}$ be the canonical map, which, by construction is a continuous *-homomorphism. Then $\tau\left(\delta_{F+C}\right)=\tau\left(\delta_{F}\right) \forall C \in \mathscr{C}$, because $\delta_{F+C}-\delta_{F}=\left(\delta_{C}-1\right) \delta_{F} \in C^{*}\left(\delta_{\mathscr{C}}-1\right) \overline{\Delta(H, B)}=\operatorname{Ker} \tau$. Define the factor map $\theta: \Delta(H, B) \mapsto \Delta(H / \mathscr{C}, \tilde{B})$ as the linear map $\theta\left(\delta_{F+C}\right)=\delta_{[F]} \forall C \in \mathscr{C}$. Then $\theta\left(\delta_{F+C_{1}}\right.$ $\left.\delta_{G+C_{2}}\right)=\delta_{[F+G]} \exp (-i / 2) B(F, G)=\delta_{[F]+[G]} \exp (-i / 2) \tilde{B}([F],[G])=\delta_{[F]} \delta_{[G]}=$ $\theta\left(\delta_{F+C_{1}}\right) \theta\left(\delta_{G+C_{2}}\right)$, and so $\theta$ is a *-homomorphism. That it is continuous in the $C^{*}$ norm, is seen from the following argument. Each $\omega \in \wp\left(\Delta_{1}(H / \mathscr{C}, \widetilde{B})\right)$ can be identified with a state $\tilde{\omega} \in \wp\left(\Delta_{1}(H, B)\right)$ simply by $\omega\left(\delta_{[F]}\right)=: \tilde{\omega}\left(\delta_{F}\right)$, and this identifies all of $\wp\left(\Delta_{1}(H / \mathscr{C}, \widetilde{B})\right)$ with the subset of $\wp(\Delta(H, B))$ consisting of those states satisfying $\omega\left(\delta_{\mathscr{C}}\right)=1$. Hence by Dixmier 2.7.1 for the enveloping $C^{*}$-norms:

$$
\begin{aligned}
\|\theta(A)\| & =\sup \left\{\omega\left(A^{*} A\right)^{1 / 2} \mid \omega \in \wp(\Delta(H / \mathscr{C}, \tilde{B}))\right\} \\
& \leqq \sup \left\{\omega\left(A^{*} A\right)^{1 / 2} \mid \omega \in \wp(\Delta(H, B))\right\}=\|A\|
\end{aligned}
$$


$\forall A \in \Delta_{1}(H, B)$. Hence $\theta$ is continuous in the $C^{*}$-norms, and can be extended: $\theta: \overline{\Delta(H, B)} \mapsto \overline{\Delta(H / \mathscr{C}, \widetilde{B})}$. Using the fact that $\theta$ is a *-homomorphism: $\theta\left(C^{*}\left(\delta_{\mathscr{G}}-\mathbf{1}\right)\right.$ $\overline{\Delta(H, B)})=C^{*}\left(\theta\left(\delta_{\mathscr{G}}-1\right)\right) \overline{\Delta(H, B)}=0$. Moreover, by $\tau\left(\delta_{F+\mathscr{E}}\right)=\tau\left(\delta_{F}\right)$, we see that $\tau(\operatorname{Ker} \theta)=0$. Hence $\operatorname{Ker} \theta=\operatorname{Ker} \tau$, and hence $\overline{\tau(\Delta(H, B))} \cong \overline{\theta(\Delta(H, B)})$, i.e. $\overline{\Delta(H, B)} / C^{*}\left(\delta_{\mathscr{C}}-\mathbf{1}\right) \overline{\Delta(H, B)} \cong \overline{\Delta(H / \mathscr{C}, \widetilde{B})}$.

Theorem 5.3. Let the symplectic form $B$ be nondegenerate on a test function space $\mathscr{M}$. Let $H \subset \mathscr{M}$ be a degenerate subspace and specify a constraint space $\mathscr{C} \subset H_{0}$. Denote the norm of $\overline{\Delta(\mathscr{M}, B)}$ by $\|\cdot\|_{a}$, and the norm of $\overline{\Delta(H, B)}$ by $\|\cdot\|_{b}$. Then there is a $C^{*}$ continuous *-homomorphism:

$$
\tilde{\tau}: \overline{\Delta(H, B)} / C_{b}^{*}\left(\delta_{\mathscr{C}}-\mathbf{1}\right) \overline{\Delta(H, B)} \rightarrow C_{a}^{*}\left(\delta_{H}\right) / C_{a}^{*}\left(\delta_{\mathscr{C}}-1\right) C_{a}^{*}\left(\delta_{H}\right) .
$$

Proof. By Manuceau 3.8[14], $\|A\|_{a} \leqq\|A\|_{b} \forall A \in \Delta_{1}(H, B)$, and hence as a mapping, $\|\cdot\|_{a}: \Delta_{1}(H, B) \mapsto \mathbb{R}_{+}$is continuous in the $\|\cdot\|_{b}$-topology, and hence can be extended as a $C^{*}$-seminorm to $\overline{\Delta(H, B)}$. Then by Dixmier $1.9 .13[10], C_{a}^{*}\left(\delta_{H}\right)=\overline{\Delta(H, B)} / \mathscr{I}$, where

$$
\mathscr{I}:=\left\{A \in \overline{\Delta(H, B)} \mid\|A\|_{a}=0,\|A\|_{b}>0\right\} \triangleleft \overline{\Delta(\overline{H, B})} .
$$

Since $\|\cdot\|_{a}$ is a $C^{*}$-norm on $C_{a}^{*}\left(\delta_{H}\right), \mathscr{I} \cap C_{a}^{*}\left(\delta_{H}\right)=\{0\}$. Let $\tau: \overline{\Delta(H, B)} \mapsto \overline{\Delta(H, B)} / \mathscr{I}=$ $C_{a}^{*}\left(\delta_{H}\right)$ be the canonical map. Then by the structures above, $\tau\left(C_{b}^{*}\left(\delta_{\mathscr{B}}-1\right)\right)=$ $C_{a}^{*}\left(\delta_{\mathscr{6}}-1\right)$, and so since $\tau$ is a homomorphism, $\tau\left(C_{b}^{*}\left(\delta_{\mathscr{C}}-\mathbf{1}\right) \Delta(H, B)\right)=C_{a}^{*}\left(\delta_{\mathscr{C}}-\mathbf{1}\right)$ $C_{a}^{*}\left(\delta_{H}\right)$. Then we can define $\tilde{\tau}$ in the logical way from $\tau$ on the algebra $\overline{\Delta(H, B) /}$ $C_{b}^{*}\left(\delta_{\mathscr{G}}-1\right) \bar{\Delta} \overline{(H, B)}$.

On using the $T$-procedure for a linear boson field, one always ends up with an algebra $\mathscr{R}=C_{a}^{*}\left(\delta_{H}\right) / C_{a}^{*}\left(\delta_{\mathscr{C}}-1\right) C_{a}^{*}\left(\delta_{H}\right)$. In the case of inner constraints, a constraint set $\mathscr{C}$ $\subset \mathscr{M}$ is specified, and we construct its "commutator" $\mathbf{p}:=\{F \in \mathscr{M} \mid B(F, \mathscr{C})=0\}$, which can be identified with $H$ above. One needs not have $\mathscr{C}=\mathbf{p}_{0}$. Henceforth omit the subscripts ' $a$ ' and ' $b$ ' denoting norm.

Corollary 5.4. Let notation be as in 5.3. Then $C^{*}\left(\delta_{H_{0}}-1\right) C^{*}\left(\delta_{H}\right)$ is a maximal ideal of $C^{*}\left(\delta_{H}\right)$ which contains $C^{*}\left(\delta_{\mathscr{C}}-\mathbf{1}\right) C^{*}\left(\delta_{H}\right)$, and hence $C^{*}\left(\delta_{H}\right) / C^{*}\left(\delta_{H_{0}}-1\right) C^{*}\left(\delta_{H}\right)$ is simple and

$$
C^{*}\left(\delta_{H}\right) / C^{*}\left(\delta_{H_{0}}-\mathbf{1}\right) C^{*}\left(\delta_{H}\right) \cong \overline{\Delta\left(H / H_{0}, \widetilde{B}\right)} .
$$

Proof. $\tilde{B}$ is nondegenerate on $H / H_{0}$, and so by Manuceau 4.24 [14], the $C^{*}$-algebra $\overline{\Delta\left(H / H_{0}, \widetilde{B}\right)} \cong \overline{\Delta(H, B)} / C^{*}\left(\delta_{H_{0}}-\mathbf{1}\right) \overline{\Delta(H, B)}$, and the isomorphism then follows from Theorem 5.3. The maximality of $C^{*}\left(\delta_{H_{0}}-1\right) C^{*}\left(\delta_{H}\right)$ follows from the fact that the factor algebra is simple, and the inclusion follows from $\mathscr{C} \subset H_{0}$.

We always assume that in nontrivial quantum mechanical systems there are noncommuting objects in the physical theory, and so $H_{0} \neq H$. Corollary 5.4 then guarantees the nontriviality of $\mathscr{R}$, and also gives the condition under which it will be simple:

Corollary 5.5. $\mathscr{R}=C^{*}\left(\delta_{H}\right) / C^{*}\left(\delta_{t}-1\right) C^{*}\left(\delta_{H}\right)$ is simple if $\mathscr{C}=H_{0}$, and conversely, if $\mathscr{R}$ 
is simple, then $\mathscr{R}=C^{*}\left(\delta_{H}\right) / C^{*}\left(\delta_{H_{0}}-\mathbf{1}\right) C^{*}\left(\delta_{H}\right)$, i.e. adding the extra elements of $H_{0}$ to $\mathscr{C}$ does not generate any larger an ideal.

Moreover, Corollary 5.4 gives the relation between the $T$-procedure and the treatment of the electromagnetic field by Carey, Gaffney and Hurst cf. [15]. In general situations, considering the desirability of a simple physical algebra, it seems natural to argue that the set of quantum constraints should be enlarged from $\mathscr{C}$ to $H_{0}$.

Return to the Gupta-Bleuler situation of before. Then $\mathscr{Q}=\left\{F \in \mathscr{M}\left|k_{\mu} f^{\mu}\right| C_{+}=0\right\}$ corresponds to $H$ above. The degenerate part of 2 consists of just the gradients:

$$
\mathscr{Q}_{0}=\left\{F \in \mathscr{M}\left|f_{\mu}\right| C_{+}=k_{\mu} f \mid C_{+}, f \in \mathscr{P}_{r}\left(\mathbb{R}^{4}\right)\right\} .
$$

As was mentioned in Sect. 4, the Maxwell equations $\left(F_{\mu v}{ }^{, v}\right)(f)$ correspond to the set $\mathscr{C}:=\left\{k_{\mu} k^{v} f_{v}(k)\left|C_{+}\right| f_{v} \in \mathscr{S}_{r}^{4}\left(\mathbb{R}^{4}\right)\right\}$, which is just $\mathscr{Q}_{0}$. Hence imposition of the Maxwell equations as constraints will result in the physical algebra:

$$
\mathscr{R}_{c}:=C^{*}\left(\delta_{\mathscr{2}}\right) / C^{*}\left(\delta_{\mathscr{C}}-\mathbf{1}\right) C^{*}\left(\delta_{\mathscr{2}}\right) \cong \overline{\Delta(\mathscr{Q} / \mathscr{C}, \tilde{B})}
$$

which is simple. Since $\mathscr{R}_{c}$ is nontrivial, this proves that $C^{*}\left(\delta_{\mathscr{C}}-\mathbf{1}\right) \notin \mathbf{1} \notin \mathscr{D}_{e} \cap \mathscr{F}$. This is the rigorous version of the usual heuristic approach to the Gupta-Bleuler theory. There Maxwells' equations are neither operator equations, nor equations on the physical state vectors, because they satisfy $\chi(x) \Phi=0$, rather than $\partial^{\mu} A_{\mu}(x) \Phi=0$. It is well known that Maxwells' equations are only satisfied as expectation values

$$
\left\langle\Phi \mid F_{\mu \nu}{ }^{, v}(x) \Phi\right\rangle=0
$$

and this is the meaning of the above considerations. $\mathscr{R}_{c}$ is also the same physical algebra as the one obtained in [15] by Carey, Gaffney and Hurst. Moreover, both $\mathscr{C}$ and 2 are Poincaré invariant, and so the Poincaré transformations are definable as automorphisms on $\mathscr{R}_{c}$ as required. Since $\mathscr{R}_{c}$ is simple, all possible constrainsts have been factored out, and as $\left\{2 k_{v} f^{\mu v} \mid f^{\mu v}=-f^{v \mu}\right\}$ corresponds to $\int F_{\mu v} f^{\mu v}$, and these are in $\mathscr{Q}$, the exponentials of the smeared field tensor are in $C^{*}\left(\delta_{2}\right)$. Hence there are nontrivial objects in $\mathscr{R}_{c}$ corresponding to the field tensor as required. This completes the algebraic side of the picture.

Finally, to obtain the full structure of the heuristic Gupta-Bleuler theory, we need to show the existence of a cyclic representation $\pi: \mathscr{F} \mapsto \mathrm{Op}(\mathscr{H})$, where $\mathscr{H}$ is an indefinite inner product space which contains a positive and bounded subspace $\mathscr{H}^{\prime}$ preserved under $\pi\left(C^{*}\left(\delta_{2}\right)\right)$, and if $\mathscr{H}^{\prime \prime}$ is the null-space of $\mathscr{H}^{\prime}$, then we must have: $\pi\left(C^{*}\left(\delta_{2}-1\right) C^{*}\left(\delta_{2}\right)\right) \mathscr{H}^{\prime} \subset \mathscr{H}^{\prime \prime}$. Moreover, $\mathscr{H}^{\prime}$ should contain the cyclic vector $\Phi_{0}$, the Poincare transformations must be quasiunitarily representable on $\mathscr{H}$, and in addition should preserve $\mathscr{H}^{\prime}, \mathscr{H}^{\prime \prime}$, and $\Phi_{0}$.

There are two approaches, both giving the same physical result, but differing in how close we remain to the heuristic situation. The Fock-type generating functional $\rho(F)=\exp (-1 / 4)(F, F)$ with $(\cdot, \cdot)$ the Gupta-Bleuler indefinite inner product: $(F, H):=-\int_{C_{+}} \frac{d^{3} k}{k_{0}} \overline{f_{\mu}(k)} h^{\mu}(k)$ would seem to be associated to the heuristic situation via a construction such as Mintchev's [16], and the correspondence $W(F) \leftrightarrow \delta_{F}$, 
where $W(F)$ denote the Weyl operators defined on $\mathscr{H}$. About $(\cdot, \cdot)$, we observe the following facts:

(i) $((\Lambda, a) F,(\Lambda, a) F)=(F, F) \forall F \in \mathscr{M}$, i.e. it is Poincaré invariant,

(ii) $(F, F) \geqq 0 \forall F \in \mathscr{Q}$, i.e. it is positive on $\mathscr{Q}$,

(iii) $(F, C)=0 \forall F \in \mathscr{Q}, C \in \mathscr{C}$, i.e. it is $\mathcal{Q}$-degenerate on $\mathscr{C}$.

Now $\rho(F)$ will define a functional $\omega_{0}$ on the *algebra $\Delta(\mathscr{M}, B)$, but this cannot be extended to either $\Delta_{1}(\mathscr{M}, B)$ or to $\mathscr{F}=\overline{\Delta(\mathscr{M}, B)}$, because it is discontinuous in these norms, cf. [17]: The sequence $\left\{(1 / n) \delta_{n F}\right\}, n \in \mathbf{N}$ converges to zero in $\|\cdot\|_{1}$, but $\left|\omega_{0}\left((1 / n) \delta_{n F}\right)\right|=(1 / n)|\rho(n F)| \rightarrow \infty$ when $n \rightarrow \infty$.

Nevertheless, because $(\cdot, \cdot)$ is positive on $\mathscr{Q}, \rho(F)$ will define a Poincaré invariant state on $C^{*}\left(\delta_{2}\right)$. As the norm of $C^{*}\left(\delta_{2}\right)$ differs from that of $\overline{\Delta(\mathcal{Q}, B)}$, we verify this last statement. $\rho$ induces a positive state $\omega_{1}$ on $\Delta_{1}(\mathscr{Q}, B)$. Now $\left|\omega_{1}\left(\delta_{F}\right)\right|=\exp ((-1 / 4)$ $(F, F)) \leqq 1=\left\|\delta_{F}\right\|_{\mathscr{F}} \forall F \in \mathcal{Q}$. Hence $\omega_{1}$ is continuous in the $\mathscr{F}$-norm, and so can be extended to a continuous functional $\omega$ on $C^{*}\left(\delta_{2}\right)$. That $\omega$ is positive and a state, follows from the fact that the extension is norm-preserving, and so $\|\omega\|=\omega(\mathbf{1})=1$, as $1 \in \Delta(\mathscr{Q}, B)$. Furthermore, from (iii) above, $(C, C)=0 \forall C \in \mathscr{C}$, and so $\omega\left(\delta_{C}\right)=1 \forall C \in \mathscr{C}$. Hence $\omega$ is a Dirac state on $C^{*}\left(\delta_{2}\right)$, and so we have $C^{*}\left(\delta_{2}\right) C^{*}\left(\delta_{2}-\mathbf{1}\right) \subset \operatorname{Ker} \omega$. Thus $\omega$ defines a Poincaré invariant state on $\mathscr{R}_{c}$, from which we can obtain a Poincaré covariant representation of $\mathscr{R}_{c}$. Since $\mathscr{R}_{c}=\overline{\Delta(\mathscr{Q} / \mathscr{C}, \widetilde{B})}$, the state defined on $\mathscr{R}_{c}$ by $\omega$ is a Fock-state. In the following two approaches below, we will retain this structure on the physical algebra, but vary the objects on the nonphysical parts of $\mathscr{F}$.

In the first approach for obtaining an indefinite inner product representation which resembles the heuristic structures, we try to remain as close to these structures as possible. In this situation, we have an indefinite hermitian functional $\omega_{0}$ only, on the *-algebra $\mathscr{F}_{0}:=\Delta(\mathscr{M}, B)$ and it is Poincaré invariant. This *-algebra contains the structures: $\Delta(\mathscr{Q}, B) \triangleright \Delta(\mathscr{Q}, B) \cdot \mathscr{A}_{0}^{*}\left(\delta_{\mathscr{G}}-1\right)$, where $\mathscr{A}_{0}^{*}(\cdot)$ denotes the $*$-algebra generated in $\Delta(\mathscr{M}, B)$ by its argument. Moreover, we have that objects corresponding to the smeared field tensor are in $\Delta(\mathscr{Q}, B)$, and objects corresponding to the smeared left-hand sides of the Maxwell equations are in $\Delta(\mathscr{Q}, B) \mathscr{A}_{0}^{*}\left(\delta_{\mathscr{C}}-\mathbf{1}\right)$. Construct the GNS-type representation of $\omega_{0}$. Let $\xi: \Delta(\mathscr{Q}, B) \mapsto \Delta(\mathscr{Q}, B) / N_{\omega_{0}}$ be the canonical map, where $N_{\omega_{0}}:=\left\{A \in \mathscr{F}_{0} \mid \omega_{0}\left(A^{*} B\right)=0 \forall B \in \mathscr{F}_{0}\right\}$. Then the linear space $\mathscr{H}=\mathscr{F}_{0} / N_{\omega_{0}}$ is a left $\mathscr{F}_{0}$-module by $A \xi_{x}=\xi_{A_{x}} \forall A, x \in \mathscr{F}_{0}$ with the natural indefinite inner product: $\left(\xi_{x}, \xi_{y}\right)_{0}:=\omega_{0}\left(x^{*} y\right)$. The cyclic element is $\xi_{1}$, and we identify

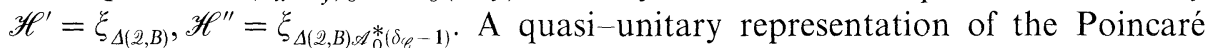
group is obtained by $U_{(\Lambda, a)}\left(\xi_{x}\right):=\xi_{(\Lambda, a) x}$ and by observing that $N_{\omega_{0}}$ is Poincaré invariant because $\omega_{0}$ is. The connection with the heuristic structures is then obtained since we have that $\mathscr{H}^{\prime}$ is positive with relation to $(\cdot, \cdot)_{0}$, because $\omega_{0}$ is positive on $\Delta(\mathscr{Q}, B), \mathscr{H}^{\prime \prime}$ is the zero-norm part of $\mathscr{H}^{\prime}$, and this follows from $\omega_{0}\left(\delta_{\mathscr{C}}-\mathbf{1}\right)=0$, and the Cauchy-Schwartz inequality for $\omega_{0}$ on $\Delta(\mathscr{Q}, B)$. Moreover, $\Delta(\mathscr{Q}, B) \mathscr{H}^{\prime}=\mathscr{H}^{\prime}, \Delta(\mathscr{Q}, B) \mathscr{A}_{0}^{*}\left(\delta_{\mathscr{G}}-1\right) \mathscr{H}^{\prime} \subseteq \mathscr{H}^{\prime \prime}$, which follows from $\Delta(\mathscr{Q}, B)$ $\mathscr{A}_{0}^{*}\left(\delta_{\mathscr{G}}-\mathbf{1}\right) \triangleleft \Delta(\mathscr{Q}, B)$, and we have a quasi-unitary representation of the Poincaré group on $\mathscr{H}$ which preserves $\mathscr{H}^{\prime}$ and $\mathscr{H}^{\prime \prime}$. The cyclic vector $\xi_{1} \in \mathscr{H}^{\prime}$.

From a mathematical point of view, the limitation to *algebras in the approach above is quite unsatisfactory in a $C^{*}$-framework, even if on the physical $C^{*}$-algebra 
$\mathscr{R}_{c}$ everything is well-defined. In this second approach, we deviate from the details of the heuristic theory on the nonphysical objects, but still obtain structures which are analogous to these for the full $C^{*}$-algebras. We observe [2], that given a situation $\mathscr{D}_{c} \triangleleft \mathcal{O}_{c} \subset \mathscr{F}$ as outlined in Sect. 2, that the GNS-type representation of any hermitian functional $f$ on $\mathscr{F}$ satisfying the conditions: $f\left(\alpha_{G}[A]\right)=f(A) \forall A \in \mathscr{F}$, $f\left(\mathcal{O}_{c+}\right) \geqq 0, f\left(\mathscr{D}_{c}\right)=0$ will have the right structures of the type needed in physics. Here $\alpha$ denoted a representation of the physical symmetry group $G$. We set out to obtain such a functional $f$ such that it coincides with the state $\omega$ induced by $\rho(F)=\exp (-1 / 4)(F, F)$ on $C^{*}\left(\delta_{2}\right)$. This is done simply by showing that $\omega$ can always be extended to a $G$-invariant hermitian functional on $\mathscr{F}$. Now $G$ acts as symplectic transformations on $\mathscr{M}$ and $\mathscr{Q} \subset \mathscr{M}$ is a $G$-invariant subspace. Then every $G$-invariant state $\varphi$ on $C^{*}\left(\delta_{2}\right)$ can be extended to a $G$-invariant state $\tilde{\varphi}$ on $C^{*}\left(\delta_{\mathscr{M}}\right)=\mathscr{F}$ by defining $\tilde{\varphi}\left(\delta_{F}\right)=0$ for $F \notin \mathscr{Q}$. Hence the $\omega$ above cannot only be extended to a $G$-invariant functional on $\mathscr{F}$, but it can indeed be extended to a $G$-invariant state $f$ on $\mathscr{F}$. This simplifies the structures previously obtained, in that from $\mathscr{D}_{c} \subset N_{f}$, we get that $\mathscr{H}=\mathscr{H}_{f}$, the latter being the GNS-representation space of $f$ with cyclic vector $\xi_{1} ; \mathscr{H}^{\prime \prime}=\mathscr{D}_{c} \xi_{1}=0, \mathscr{H}^{\prime}=C^{*}\left(\delta_{2}\right) \xi_{1}=\mathscr{H}_{\text {phys }}$ and $U_{(\Lambda, a)}\left(\xi_{x}\right):=\xi_{(\Lambda, a) x}$ as before. Hence $\mathscr{H}^{\prime}$ can be characterized as the subspace of $\mathscr{H}$ consisting of all vectors annihilated by the supplementary conditions, just as is done in heuristic physics for the physical subspace. So in this general situation where we consider the full $C^{*}$-algebras, there is no need to use indefinite inner product representations. We do know however that none of these positive representations can have a Fock structure, which is seen from the result by Barut and Raczka [20], that the zero mass representation of the Poincare group on a space of tensor valued functions must necessarily have indefinite metric. Hence if one wants to find Poincare covariant Fock representations, one is forced to look for these in the set of nonpositive extensions of $\omega$ to $G$-invariant hermitian functionals on $\mathscr{F}$. There is no guarantee that such functionals exist.

Since both representation methods above still produce the same representation for the physical $C^{*}$-algebra $\mathscr{R}_{c}$, there is nothing to choose between them from a physical point of view.

We summarize the final indefinite inner product (IIP) representation structures. There is a state $\omega$ on $C^{*}\left(\delta_{2}\right)$ such that $\omega\left(\delta_{F}\right)=\exp (-1 / 4)(F, F), \mathscr{D}_{c}=$ $C^{*}\left(\delta_{\mathscr{Q}}\right) C^{*}\left(\delta_{\mathscr{C}}-\mathbf{1}\right) \subset \operatorname{Ker} \omega, \omega\left(\alpha_{G}(A)\right)=\omega(A)$. This induces a Fock-state on $\mathscr{R}_{c}$.

Outside $\mathcal{O}_{c}$, we considered two structures on $\mathscr{F}$, first, on the *-algebra $\mathscr{F}_{0}=\Delta(\mathscr{M}, B)$ we extend $\omega$ to $\omega_{0}$ given by $\omega_{0}\left(\delta_{F}\right)=\exp (-1 / 4)(F, F)$. It is discontinuous in the $C^{*}$-norm and so cannot be extended to $\mathscr{F}$. Then $\omega_{0}$ is Poincare invariant, and its GNS-representation has the right IIP-structure. We conjecture that it will also have a Mintchev Fock-structure.

Second, we extended the state $\omega$ to a state $f$ on $\mathscr{F}$ such that it is Poincare invariant. The GNS-representation of $f$ has also the right structures, but now for the full $C^{*}$-algebras, not only the ${ }^{*}$-algebras contained in $\mathscr{F}_{0}$. However, $f$ differs from the heuristic structures outside $\mathcal{O}_{c}$, and it will not have a Fock-structure.

At this point the indispensability of the IIP for degenerate systems can be discussed, for it becomes clear that any Dirac state on $\mathcal{O}_{c}$ in the present model can be extended to a $G$-invariant state on $\mathscr{F}$, but its GNS-representation will not have 
a Fock structure. If Fock structures are obtainable at all for the full $C^{*}$-algebras, these will be associated with nonpositive extensions of the Dirac states on $\mathcal{O}_{c}$.

\section{Conclusions}

In this paper, we have shown the rigorous treatment of the free Gupta-Bleuler electromagnetic field to be divided into two stages. The first stage establishes a suitable $C^{*}$-algebra formulation for the electromagnetic field when only the positive frequency constraint is involved. This requires an extension of the theory of quantization of linear fields in order to treat correctly the use of quadratic expressions $\chi^{*}(f) \chi(f)$, albeit smeared. The second stage is the construction of a representation of this $C^{*}$-algebra theory on an indefinite inner product space.

The abstract algebraic theory of the Gupta-Bleuler field would be rounded off by showing the Poincaré transformations to be inner on $\mathscr{R}_{c}$. This was done by Carey and Hurst for the Fermi-gauge in [18], but since the demonstration of it falls outside the scope of this article, we leave that for a future publication.

Clearly the type of situation which can be described by the preceding abstract theory of outer constraints is limited by our ability to define the outer constraints as automorphisms on the field algebra. For the above situation of $\mathscr{F}=\overline{\Delta(\mathscr{M}, B)}$ with correspondences $\delta_{F} \leftrightarrow \exp i A(F)$, the constraints $\chi(H)$ should at least let $\log \left(e^{i \chi(H)} e^{i A(F)} e^{-i \chi(H)}\right)$ be an object linear in $A$. This means that quadratic constraints can be treated, but higher degree constraints many still be only amenable in the concrete situation.

The algebraic methods above allow us to set up quadratic field theories in a constructive way in a $C^{*}$-framework. That is, starting from the $C^{*}$-algebra of the CCR, $\mathscr{F}_{l}=\overline{\Delta(\mathscr{M}, B)}$ for the linear fields, we define specified quadratic fields as automorphisms on $\mathscr{F}_{l}$ as above. The automorphisms need not commute, and will define a group $G \subset$ Aut $\mathscr{F}_{l}$. Since the smearing of the quadratic fields will give locally compactness problems as above, $G$ will not be locally compact, but will be an inductive limit of locally compact groups. Hence as before, we can obtain:

$$
\mathscr{F}:=\overline{\bigcup_{s \in I} \mathscr{A}^{(s)}}, \quad \text { where } \quad \mathscr{A}^{(s)}:=C^{*}\left(\mathscr{F}_{l} \cup U\left(G_{s}\right)\right) \text {. }
$$

$I$ is the directed index set, $G_{s}$ is locally compact for each $s \in I, G_{s} \subseteq G_{p}$ if $s \leqq p$. Then $\mathscr{F}$ can be chosen as the new field algebra of the given quadratic fields. Unfortunately, it is not possible to define higher powers of fields as automorphisms on $\mathscr{F}$, as a glance at the $\mathrm{CBH}$-formula makes clear.

\footnotetext{
Acknowledgements. My warm and special thanks is due to Professor C. A. Hurst. I am especially grateful to him for directing my attention to, and stressing the importance of the locally compactness problem, for providing many elucidating remarks to this paper, and for having been a constant source of interested and illuminating feedback in regard to this problem. It was also Prof. Hurst who provided the opportunity for this work. I have also benefitted from many helpful discussions with Prof. W. Moran, Dr. Alan Carey, and the ghost of Janice Gaffney. Finally, I would like to thank one of the refee to this paper for pointing out flaws in the reasoning of an earler version of [2], and for providing the positive extension of $\omega$ in Sect. 5 .
} 


\section{References}

1. Grundling, H. B. G. S., Hurst, C. A.: Algebraic quantisation of systems with a gauge degeneracy. Commun. Math. Phys. 98, 369-390 (1985)

2. Grundling, Hendrik, Hurst, C. A.: Algebraic structures of degenerate systems and the indefinite metric. J. Math. Phys. 28, 559-572 (1987)

3. Grundling, Hendrik: Algebraic structures of degenerate systems. Adelaide Unıversity, Ph.D. thesis 1986

4. Emch, G. G.: Algebraic methods in statistical mechanics and quantum field theory. New York: Wiley 1972

5. Dirac, P. A. M.: Lectures in quantum mechanics. New York: Belfer Graduate School of Science, Yeshiva University 1964; Dirac, P. A. M.: Generalised Hamiltonian dynamics. Can. J. Math. 2, 129-148 (1950); Dirac, P. A. M.: The Hamiltonian form of field dynamics. Can. J. Math. 3, 1-23 (1951)

6. Carey, A. L., Hurst, C. A.: A note on the Fermi quantisation of Landau gauge. Lett. Math. Phys. 2, 227-234 (1978)

7. Manuceau, J.: $C^{*}$-algebre de relations de commutation. Ann. Inst. Henri Poincaré 8, 139-161 (1968)

8. Pedersen, G. K.: $C^{*}$-algebras and their automorphism groups. London: Academic Press 1979

9. Green, P.: The local structure of twisted covariance algebras. Acta Math. 140, 191-250 (1978)

10. Dixmier, J.: $C^{*}$-algebras. Amsterdam: North Holland 1977

11. Gupta, S. N.: Theory of longitudinal photons in quantum electrodynamics. Proc. Phys. Soc. Lond. A. 63, 681-691 (1950); Bleuler, K.: Eine neue Methode zur Behandlung der longitudinalen und skalaren Photonen. Helv. Phys. Acta 23, 567-586 (1950)

12. Strocchi, F., Wightman, A. S.: Proof of the charge superselection rule in local relativistic quantum field theory. J. Math. Phys. 15, $2198-2224$ (1974); Strocchi, F., Wightman, A. S.: Erratum: Proof of the charge superselection rule. J. Math. Phys. 17, 1930-1931 (1976)

13. Takeda, Z.: Inductive limit and infinite direct product of operator algebras. Tohoku Math. J. 7, 68-86 (1955)

14. Manuceau, J., Sirugue, M., Testard, D., Verbeure A.: The smallest $C^{*}$-algebra for canonical communication relations. Commun. Math. Phys. 32, 231-243 (1973)

15. Carey, A. L., Gaffney, J. M., Hurst, C. A.: A $C^{*}$-algebra formulation of the quantisation of the electromagnetic field. J. Math. Phys. 18, 629-640 (1977)

16. Mintchev, M. Quantisation in indefinite metric. J. Phys. A. 13, 1841-1859 (1980)

17. Jakobczyk, L.: Canonical quantisation with indefinite inner product. Ann. Phys. 161, 314-336 (1985)

18. Carey, A. L., Hurst, C. A.: The Fermi method of quantizing the electromagnetic field as a model for quantum field theory. J. Math. Phys. 18, 1553-1561 (1977)

19. Akemann, C. A., Pedersen, G. K., Tomiyama, J.: Multipliers of $C^{*}$-algebras. J. Funct. Anal. 13. 277-301 (1973)

20. Barut, A. O., Raczka, R.: Properties of non-unitary zero mass induced representations of the Poincaré group on the space of tensor-valued functions. Ann. Inst. H. Poincaré 17, 111-118 (1972)

Communicated by H. Araki

Received September 22, 1986; in revised form April 16, 1987 
